This article was published in an Elsevier journal. The attached copy

is furnished to the author for non-commercial research and education use, including for instruction at the author's institution, sharing with colleagues and providing to institution administration.

Other uses, including reproduction and distribution, or selling or licensing copies, or posting to personal, institutional or third party websites are prohibited.

In most cases authors are permitted to post their version of the article (e.g. in Word or Tex form) to their personal website or institutional repository. Authors requiring further information regarding Elsevier's archiving and manuscript policies are encouraged to visit: 


\title{
Dynamic analysis of beam structures considering geometric and constitutive nonlinearity
}

\author{
P. Mata, S. Oller *, A.H. Barbat \\ Technical University of Catalonia, UPC, Edificio Cl, Campus Nord, Gran Capitá s/n, 08034 Barcelona, Spain
}

Received 30 October 2006; received in revised form 21 September 2007; accepted 24 September 2007

Available online 29 September 2007

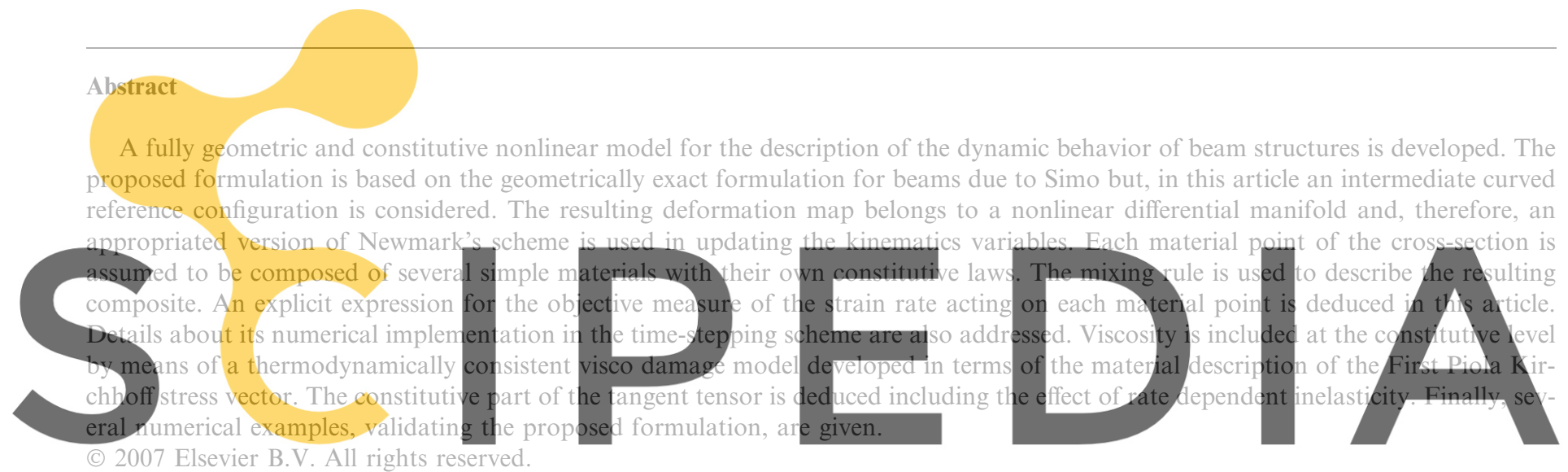

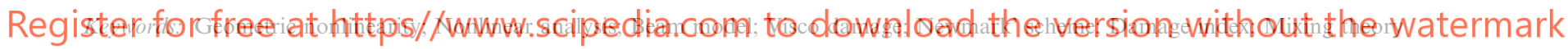

\section{Introduction}

Several works performing the nonlinear dynamic analysis of beam structures have been focused on the formulation of geometrically consistent models considering finite displacements and rotations and using simplified linear constitutive relations for the cross-sectional forces [18]. Most of the recent works invoke the formulation and the numerical implementation proposed by Simo and Simo and Vu-Quoc [47-49], which generalize the formulation developed by Reissner $[42,43]$ to the dynamic case. This formulation employs a director type approach when describing the configuration of the beam cross-sections during the motion, considering finite shearing and finite extension, as described by Antman [1]. The works of Simo

\footnotetext{
* Corresponding author. Tel.: +34 934017401; fax: +34934011048.

E-mail address: sergio.oller@upc.edu (S. Oller).
}

and Vu-Quoc consider a straight and unstressed rod as reference configuration and the hypothesis of plane cross-sections. The resulting deformation map can be identified with elements belonging to the differential manifold obtained from the rotation group $S O(3)$ and the canonical (linear) vector space $\mathbb{R}^{3}$, i.e. $S O(3) \times \mathbb{R}^{3}$. A detailed discussion about finite rotations can be consulted in Ref. [2].

Posteriorly, Ibrahimbegović extends the formulation given in Ref. [47] considering a curved reference configuration of the rods [19]. Other authors develop equivalent formulations starting from different physical principles [30,31] and obtaining advantages over the original formulation of Simo and Vu-Quoc, such as avoiding the shear locking in the numerical simulations [27] or ensuring the invariance of the continuum problem [12,24,52]. Additionally, a great number of applications of these models are available for studying, e.g. the bifurcation points, the buckling behavior of structures $[10,22,25]$ or the trajectory of flexible mechanisms involving large rotations $[21,23]$. 
An important effort has been devoted to develop timestepping schemes for the integration of the nonlinear dynamic equations of motion involving finite rotations. The main difficulty arises in the fact that the deformation map takes values in the differentiable manifold $S O(3) \times \mathbb{R}^{3}$ and not in a linear space, as it is the case in classical dynamics. An implicit time-stepping algorithm is developed in Ref. [49] extending the classical Newmark's scheme to the rotation group $S O(3)$, obtaining a formulation similar to that of the linear case. In the same work, the consistent linearization of the weak form of the balance equations yields to a tangential inertia tensor, nonsymmetric in the rotational components. A comparison among implicit time stepping schemes according to different choices of rotational parameters can be reviewed in Ref. 「261.

Even when Newmark's scheme has been widely applied to the study of the dynamic response of structures, rigid bodies and flexible mechanisms, Mäkinen [34] states that it only constitutes an approximated version of the corrected formulae, which are given in his work for the spatial and material descriptions. The main reasons are that material descriptions of the spin and acceleration vectors involved in the updating procedures belong to different tan-

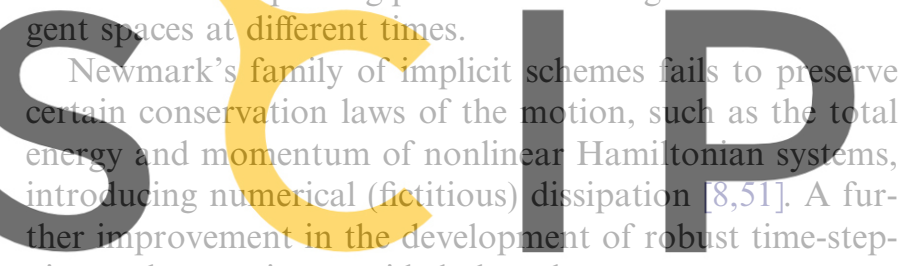
ping schemes is provided by the energy-momentum

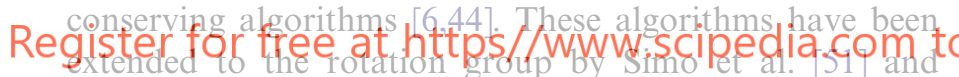
applied to the nonlinear dynamic problems of rods, shells and rigid bodies. In Refs. [20,23], a complete study of the general dynamics of flexible mechanisms is carried out and a conserving/decaying time-stepping scheme is proposed for eliminating the high frequency content in the response of flexible structures.

As it has been detailed by the authors in Ref. [35], the simulation of constitutive nonlinearity in beams has been developed by means of concentrated and distributed models [40] and, in most cases, the geometric nonlinearity has been neglected. Only a few recent works consider fully consistent geometric and constitutive nonlinear formulations but, they have been mainly focused on plasticity [45] and on the static analysis of the structures $[15,53]$. It is worth noting that Ref. [50] is one of the few works that considers time dependent effects in the formulation of a geometrically exact beam model including visco plasticity.

Recently, Mata et al. [35] proposed a suitable formulation for both geometric nonlinearity and a general distribution of composite materials on the cross-sections of the beams. The proposed fiber-like formulation [46] takes into account thermodynamically consistent three-dimensional (3D) constitutive laws for each simple material [16] and the simple mixing rule to treat the resulting composite
[39]. The tangential tensors of the beam are derived starting from the linearization of the constitutive relations at material point level. The resulting discrete system of equations is solved using the stiffness displacement-based procedure [40] in the context of the finite element method (FEM). Therefore, several elements are required to predict realistically the behavior of complex structures. The numerical solution is affected by strain localization for materials with softening behavior. A technique based on the regularization of the energy dissipated at any material point ensures that the whole structural response remains objective $[7,16]$, but the length of the zone where softening occurs is still mesh dependent [36]. In any case, the model of Ref. [35] is able to handle only static (cyclic or not) loading paths.

This article in an extension to the dynamic case of the work presented in Ref. [35]. Details about the kinematics of the geometrically exact formulation of beams considering an initially curved reference configuration, on the introduction to large rotations, and on the specific constitutive equations, can be consulted in Refs. [19,35,48].

The contributions of this work are the following: (i) An explicit expression is deduced for the objective measure of the strain rate existing on each material point of the cross-

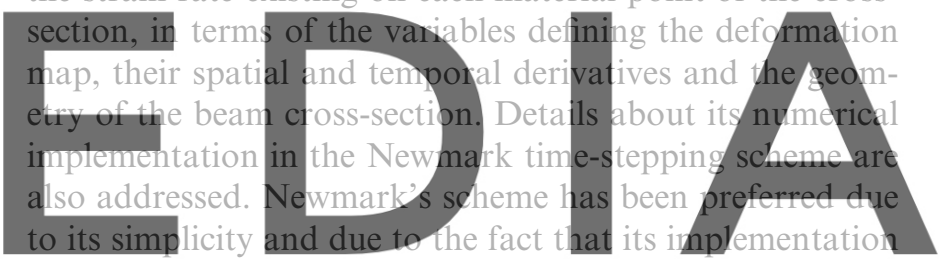

in a standard FE code is straightforward, even though the

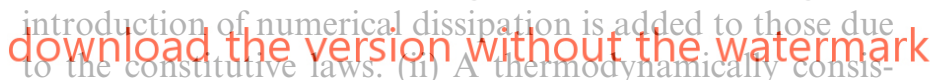

tent visco damage model, developed in terms of the material description of the First Piola Kirchhoff (FPK) stress vector acting on each material point of the cross-section is described and included into the mixing theory for composite materials. In this way, viscosity is considered at constitutive level. (iii) Starting from the linearization of the weak form of the balance equations, the constitutive part of the tangent tensor is deduced, including the effect inelasticity. The rate dependent part of the constitutive equations allows deducing a consistent tangent viscous stiffness. Finally, several numerical examples are shown, validating the proposed formulation. Several practical aspects of the performance of a engineering structure are considered: distribution of the damage, overstrength and ductility.

\section{Strain and strain rates for initially curved beams}

The formulation of Simo and Vu Quoc [47,48] for beams that can undergo large deformations considering a free of stress curved reference configuration has been already presented in [35], but, previous works can be consulted $[19,30,31]$. Only a brief summary will be given here for introducing the calculation of the strain rate measurements. 


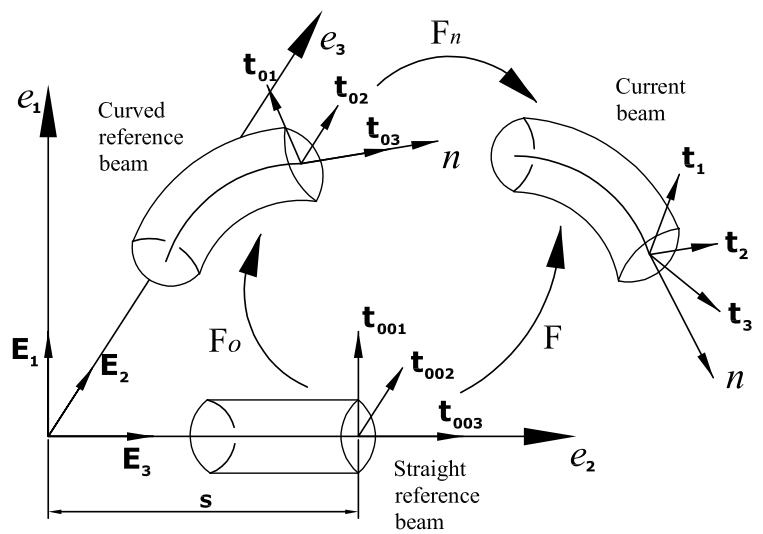

Fig. 1. Configurational description of the beam model.

Given $\left\{\widehat{E}_{i}\right\}$ and $\left\{\hat{e}_{i}\right\}$ the fixed ${ }^{1}$ (inertial) material and spatial frames, respectively, the position vector of any material point on the straight reference beam is $\widehat{X}=\xi_{i} \widehat{E}_{i}$, where $\xi_{\beta}$ are coordinates directed along $\left\{\widehat{E}_{\beta}\right\}$ describing points on the cross-section. The curved reference beam is defined by the fixed curve $\hat{\varphi}_{0 i}=\hat{\varphi}_{0}(S) \hat{e}_{i} \in \mathbb{R}^{3}$, where $S \equiv \xi_{1} \in[0, L]$ is the arch-length parameter. Each point on this curve has rigidly attached an orthonormal local

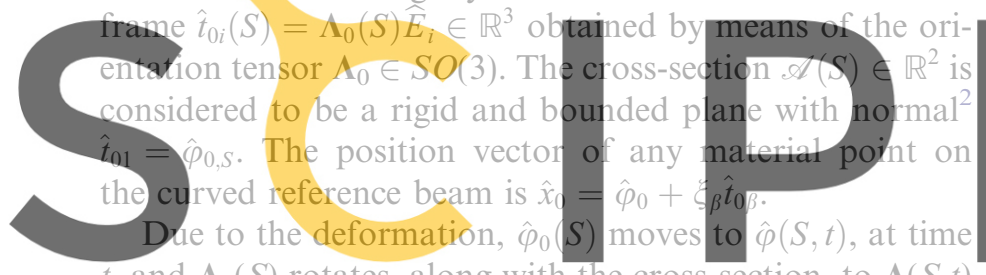

$t$, and $\Lambda_{0}(S)$ rotates, along with the cross-section, to $\Lambda(S, t)$ by means of the incremental rotation tensor $\Lambda_{n}$, i.e. $\Lambda=$

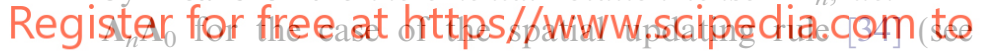

Fig. 1). In general, the normal vector $\hat{t}_{1}$ does not coincides with $\hat{\varphi}, S$ because of the shearing [18]. The current position vector of any material point on the moving beam is $\hat{x}=$ $\hat{\varphi}+\xi_{\beta} \hat{t}_{\beta}$. The current cross-section configuration is completely determined by the pair $(\hat{\varphi}, \Lambda) \in \mathbb{R}^{3} \times S O(3)$ [48].

The spatial placement of the beam is defined as $\mathscr{B}_{t}:=$ $\left\{\hat{x} \in \mathbb{R}^{3} \mid \hat{x}=\hat{\varphi}+\xi_{\beta} \hat{t}_{\beta}\right\}$ and its tangent space is $T_{\hat{x}} \mathscr{B}_{t}:=$ $\left\{\delta \hat{x} \in \mathbb{R}^{3} \mid \hat{x} \in \mathscr{B}_{t}\right\}$ for any spatial kinematically admissible variation $^{3} \quad \delta \hat{x} \approx(\delta \hat{\varphi}, \delta \hat{\theta}) \in \mathbb{R}^{3} \times T_{\Lambda}^{\text {spa }} \quad$ so that $\delta \boldsymbol{\Lambda}=$ $(\delta \hat{\theta} \times \boldsymbol{\Lambda}) \in T_{\Lambda}^{\mathrm{spa}} S O(3)$.

The deformation gradients for the curved reference and current beam configurations relative to the straight reference beam, $\mathbf{F}_{0}$ and $\mathbf{F}$, can be consulted in Ref. [35]. The

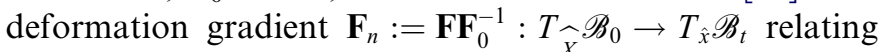
the curved reference configuration with the current placement is directly responsible for the development of strains, its explicit expression is given by [30]

\footnotetext{
${ }^{1}$ The indices $i$ and $\beta$ range over $\{1,2,3\}$ and $\{2,3\}$, respectively.

2 The symbol $(\bullet),{ }_{x}$ denotes the partial differentiation of $(\bullet)$ with respect to $x$.

${ }^{3}$ The symbol $T_{\Lambda}^{\text {spa }} S O(3)$ denotes the spatial form of the tangential space to $\mathrm{SO}(3)$, with base point $\Lambda$, and $T_{\Lambda}^{\mathrm{spa}}$ is the spatial linear vector space of rotations, with base point $\boldsymbol{\Lambda}$, as described in Ref. [33].
}

$\mathbf{F}_{n}=\frac{1}{\left|\mathbf{F}_{0}\right|}\left[\hat{\varphi}_{, S}-\boldsymbol{\Lambda}_{n} \hat{\varphi}_{0, S}+\tilde{\boldsymbol{\omega}}_{n} \xi_{\beta} \hat{t}_{\beta}\right] \otimes \hat{t}_{01}+\boldsymbol{\Lambda}_{n}$,

where $\left|\mathbf{F}_{0}\right|$ is the determinant of $\mathbf{F}_{0}$ and $\tilde{\boldsymbol{\omega}}_{n}=\boldsymbol{\Lambda}_{n, S} \boldsymbol{\Lambda}_{n}^{\mathrm{T}}$ $\in T_{\Lambda}^{\mathrm{spa}} S O(3)$ is the current curvature tensor relative to the curved reference beam. The material representation of $\mathbf{F}_{n}$ is $\mathbf{F}_{n}^{\mathrm{m}}=\boldsymbol{\Lambda}^{\mathrm{T}} \mathbf{F}_{n} \boldsymbol{\Lambda}_{0}$.

Removing the rigid body component from Eq. (1), it is possible to construct the spatial strain tensor $\boldsymbol{\varepsilon}_{n}=\mathbf{F}_{n}-\boldsymbol{\Lambda}_{n}$. The associated spatial strain vector acting on a material point on the current beam cross-section is obtained as

$\hat{\varepsilon}_{n}=\boldsymbol{\varepsilon}_{n} \hat{t}_{01}=\frac{1}{\left|\mathbf{F}_{0}\right|}\left[\hat{\gamma}_{n}+\hat{\omega}_{n} \times \xi_{\beta} \hat{t}_{\beta}\right]$,

where $\hat{\gamma}_{n}=\left(\hat{\varphi}_{, S}-\hat{t}_{1}\right)$ measures elongation and shearing $[19,47]$ and $\hat{\omega}_{n} \in T_{\Lambda}^{\mathrm{spa}}$ is the current curvature vector relative to the curved reference beam corresponding to the axial vector of $\tilde{\omega}_{n}$. The material version of $\hat{\varepsilon}_{n}$ is given by

$\widehat{\mathscr{E}}_{n}=\boldsymbol{\Lambda}^{\mathrm{T}} \widehat{\varepsilon}_{n}=\left|\mathbf{F}_{0}\right|^{-1}\left[\hat{\Gamma}_{n}+\widehat{\boldsymbol{\Omega}}_{n} \times \xi_{\beta} \widehat{E}_{\beta}\right]$

where $\widehat{\Gamma}_{n}=\left(\boldsymbol{\Lambda}^{\mathrm{T}} \hat{\varphi}_{, S}-\widehat{E}_{1}\right)$ and $\widehat{\boldsymbol{\Omega}}_{n} \in T_{\Lambda}^{\mathrm{mat}}$ is the material description of the current curvature vector corresponding to the axial vector of $\widetilde{\boldsymbol{\Omega}}_{n}=\boldsymbol{\Lambda}^{\mathrm{T}} \tilde{\boldsymbol{\omega}}_{n} \mathbf{\Lambda} \in T_{\boldsymbol{\Lambda}}^{\mathrm{mat}} S O(3)$ [33].

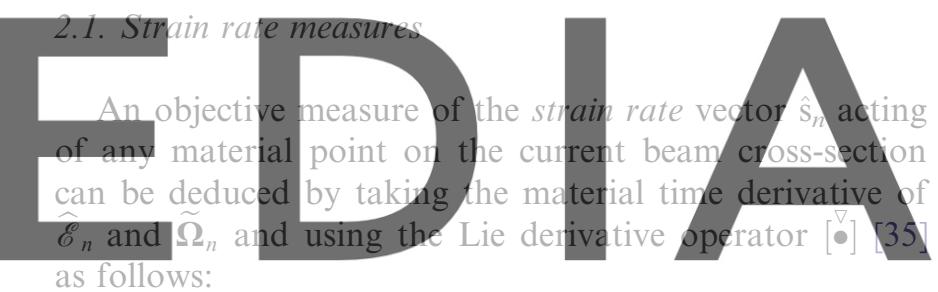

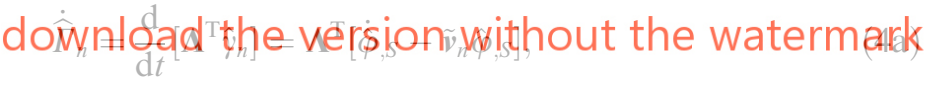

$\dot{\widetilde{\Omega}}_{n}=\frac{\mathrm{d}}{\mathrm{d} t}\left[\Lambda^{\mathrm{T}} \widetilde{\omega}_{n} \Lambda\right]=\Lambda^{\mathrm{T}} \widetilde{v}_{n, S} \Lambda=\widetilde{\mathbf{V}}_{n, S}, \quad \dot{\widehat{\Omega}}_{n}=\widehat{V}_{n, S}$,

$\left[\dot{\hat{\gamma}}_{n}\right]=\boldsymbol{\Lambda} \dot{\hat{\Gamma}}_{n}=\dot{\hat{\varphi}}_{, S}-\tilde{\boldsymbol{v}}_{n} \hat{\varphi}_{, S}$

$\left[\dot{\tilde{\boldsymbol{\omega}}}_{n}\right]=\boldsymbol{\Lambda} \dot{\widetilde{\boldsymbol{\Omega}}}_{n} \boldsymbol{\Lambda}^{\mathrm{T}}=\tilde{\boldsymbol{v}}_{n, S}, \quad\left[\dot{\hat{\hat{\omega}}}_{n}\right]=\hat{v}_{n}, S$,

where $\widetilde{\mathbf{V}}_{n}=\boldsymbol{\Lambda}_{n}^{\mathrm{T}} \dot{\boldsymbol{\Lambda}}_{n} \in T_{\boldsymbol{\Lambda}}^{\mathrm{mat}} S O(3)$ and $\tilde{\boldsymbol{v}}_{n}=\dot{\boldsymbol{\Lambda}}_{n} \boldsymbol{\Lambda}_{n}^{T} \in T_{\boldsymbol{\Lambda}}^{\text {spa }} S O(3)$ are the material and spatial forms of the current (skewsymmetric) angular velocity tensors referred to the curved reference beam and, $\widehat{V}_{n} \in T_{\Lambda}^{\text {mat }}$ and $\hat{v}_{n} \in T_{\Lambda}^{\text {spa }}$ are their corresponding axial vectors. Finally, the co-rotated strain rate vector $\hat{s}_{n}=\Lambda \widehat{\mathscr{S}}_{n}$ is obtained from Eqs. (4a)-(4d) as

$\widehat{\mathscr{S}}_{n}=\boldsymbol{\Lambda}^{\mathrm{T}} \dot{\hat{\varphi}}_{, S}-\widetilde{\mathbf{V}}_{n} \boldsymbol{\Lambda}^{\mathrm{T}} \hat{\varphi}_{, S}+\dot{\widetilde{\mathbf{\Omega}}}_{n} \xi_{\beta} \widehat{E}_{\beta}$,

$\hat{\boldsymbol{s}}_{n}=\left[\begin{array}{l}\nabla \\ {\left[\hat{\hat{\varepsilon}}_{n}\right.}\end{array}\right]=\left[\begin{array}{l}\nabla \\ {\left[\hat{\hat{\gamma}}_{n}\right.}\end{array}\right]+\left[\dot{\tilde{\boldsymbol{\omega}}}_{n}\right] \xi_{\beta} \hat{t}_{\beta}=\dot{\hat{\varphi}}_{, S}-\tilde{\boldsymbol{v}}_{n} \hat{\varphi}_{, S}+\tilde{\boldsymbol{v}}_{n, S} \xi_{\beta} \hat{t}_{\beta}$.

Eq. (5b) corresponds to the objective strain rate vector measured by an observer located on the current frame $\left\{\hat{t}_{i}\right\}$.

\subsection{Stress vector and cross-sectional stress resultants}

The strain vector $\hat{\varepsilon}_{n}$ is the energetically conjugated strain measure to the asymmetric First Piola Kirchhoff (FPK) 
stress vector [47,30], which can be defined as $\widehat{P}_{1}=P_{1 j} \hat{t}_{j} \in T_{\hat{x}}^{*} \mathscr{B}_{t}$, where $P_{1 j}$ is the component of the stress vector acting in the direction $j$ of the local frame attached to the current cross-section relative to the direction $\hat{t}_{0 j}$ in the corresponding curved reference configuration. The material description of $\widehat{P}_{1}$ is $\widehat{P}_{1}^{\mathrm{m}}=\boldsymbol{\Lambda}^{\mathrm{T}} \widehat{P}_{1}$. The cross-sectional stress resultant and the stress couple vectors ${ }^{4}$ can be estimated according to

$$
\begin{aligned}
& \hat{n}(S)=\int_{\mathscr{A}} \widehat{P}_{1} \mathrm{~d} \mathscr{A} \in T_{\hat{x}}^{*} \mathscr{B}_{t} ; \\
& \hat{m}(S)=\int_{\mathscr{A}}(\hat{x}-\hat{\varphi}) \times \widehat{P}_{1} \mathrm{~d} \mathscr{A} \in T_{\hat{x}}^{*} \mathscr{B}_{t} .
\end{aligned}
$$

The corresponding material descriptions of $\hat{n}$ and $\hat{m}$ are $\hat{n}^{\mathrm{m}}=\Lambda^{\mathrm{T}} \hat{n}$ and $\hat{m}^{\mathrm{m}}=\Lambda^{\mathrm{T}} \hat{m}$, respectively.

\subsection{Virtual work principle}

The spatial form of the reduced balance equations of the current beam, referred to the curved reference beam, can be
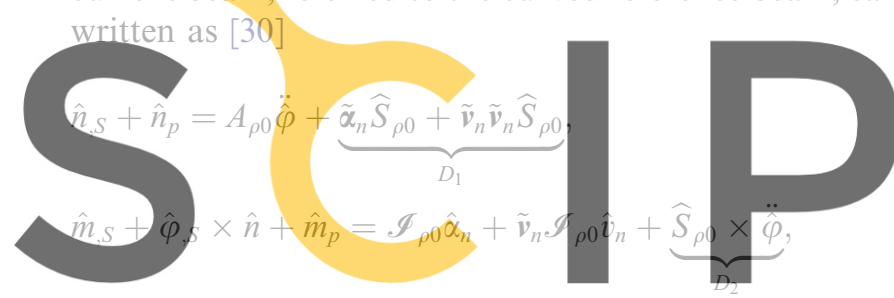

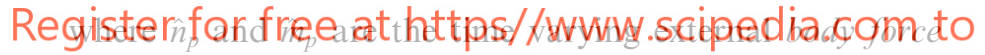
and body moment per unit of reference length, respectively; $\tilde{\alpha}_{n}=\dot{\tilde{v}}_{n} \in T_{\mathrm{A}}^{\mathrm{spa}} S O(3)$ is the spatial form of the current angular acceleration tensor referred to the curved reference beam and $\hat{\alpha}_{n} \in T_{\Lambda}^{\text {spa }}$ is its corresponding axial vector. $A_{\rho 0}$, $\widehat{S}_{\rho 0}$ and $\mathscr{I}_{\rho 0}$ are the mass density, the first mass moment density and the second mass moment density per unit of length of the curved reference beam, respectively; their explicit expressions can be consulted in Refs. [30,48]. The terms $D_{1}$ and $D_{2}$ appear due to the initial curvature and an inhomogeneous distribution of the material properties. However, for most of the practical cases of engineering structures, their contribution to the dynamics can be neglected or added to the external forces and moments.

Considering a kinematically admissible variation of the pair $(\hat{\varphi}, \boldsymbol{\Lambda})$, i.e. $h=(\delta \hat{\varphi}, \delta \hat{\theta}) \in \mathbb{R}^{3} \times T_{\Lambda}^{\text {spa }}$ [48], taking the dot product of $h$ with Eqs. (7) and (8), integrating over the length of the curved reference beam and integrating by parts for the terms $\hat{n}_{S S}$ and $\hat{m}_{, S}$, one may obtain the following nonlinear functional, $\mathbf{G}(\hat{\varphi}, \boldsymbol{\Lambda}, h)$, corresponding to the spatial description of the virtual work principle:

\footnotetext{
${ }^{4}$ The symbol '*, of $T_{\hat{x}}^{*} \mathscr{B}_{t}$ has been used to indicate that the reduced stress measures belong to the dual space of $T_{\hat{x}} \mathscr{B}_{t}$.
}

$$
\begin{aligned}
\mathbf{G}(\hat{\varphi}, \boldsymbol{\Lambda}, h)= & \underbrace{\int_{L}\left[\left(\delta \hat{\varphi}_{, S}-\delta \hat{\theta} \times \hat{\varphi}_{, S}\right) \cdot \hat{n}+\delta \hat{\theta}_{, S} \cdot \hat{m}\right] \mathrm{d} S}_{\mathbf{G}_{\text {int }}(\hat{\varphi}, \boldsymbol{\Lambda}, h)} \\
& +\underbrace{\int_{L}\left[\delta \hat{\varphi} \cdot A_{\rho_{0}} \ddot{\hat{\varphi}}+\delta \hat{\theta} \cdot\left(\mathscr{I}_{\rho_{0}} \hat{\alpha}_{n}+\tilde{\boldsymbol{v}}_{n} \mathscr{I}_{\rho_{0}} \hat{v}_{n}\right)\right] \mathrm{d} S}_{\mathbf{G}_{\text {ine }}(\hat{\varphi}, \boldsymbol{\Lambda}, h)} \\
& -\underbrace{\int_{L}\left[\delta \hat{\varphi} \cdot \hat{n}_{p}+\delta \hat{\theta} \cdot \hat{m}_{p}\right] \mathrm{d} S}_{\mathbf{G}_{\text {ext }}(\hat{\varphi}, \boldsymbol{\Lambda}, h)} \\
& -\left[\left.(\delta \hat{\varphi} \cdot \hat{n})\right|_{0} ^{L}+\left.(\delta \hat{\theta} \cdot \hat{m})\right|_{0} ^{L}\right]=0 .
\end{aligned}
$$

The functional $\mathbf{G}$ is the sum of the internal, inertial and external contributions, $\mathbf{G}_{\text {int }}, \mathbf{G}_{\text {ine }}$ and $\mathbf{G}_{\text {ext }}$, respectively. It also corresponds to the weak form of the balance equations $[19,48]$. Noting that $\Lambda_{0}$ is a fixed frame, we have that $\delta \hat{\theta}=\delta \hat{\theta}_{n}$ and, therefore, the terms $(\delta \hat{\varphi}, S-\delta \hat{\theta} \times \hat{\varphi}, S)$ and $\delta \hat{\theta}_{, S}$ correspond to the co-rotated variations of the reduced strain measures $\hat{\varepsilon}_{n}$ and $\hat{\omega}_{n}$ relative to the curved reference beam, $\delta\left[\hat{\gamma}_{n}\right]$ and $\delta\left[\hat{\omega}_{n}\right]$, respectively.

\section{Rate dependent constitutive models}

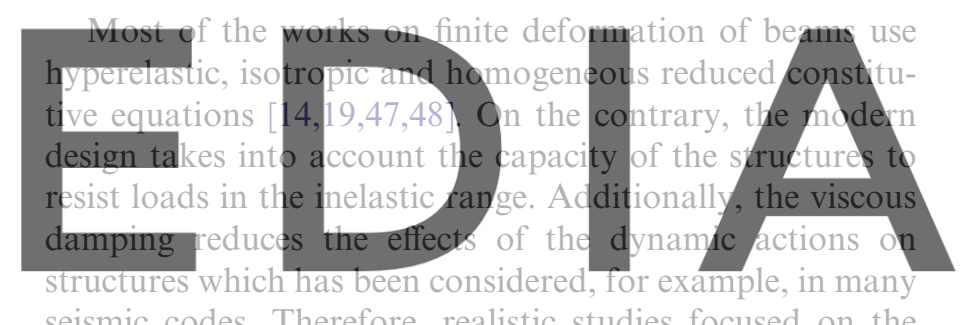
seismic codes. Therefore, realistic studies focused on the

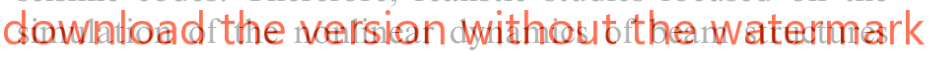
should consider rate dependent constitutive relations, as well as geometric effects. In this section, the rate independent damage model developed in [35] is extended to consider viscosity and the same formulation can be directly applied to visco elasticity. For the case of visco plastic materials, Ref. [50] can be consulted and, therefore, those results are omitted here.

The material points belonging to the cross-section are considered as composed by a homogeneous mixture of several simple materials, each of them with its own constitutive law (see Fig. 2). The mechanical behavior of the

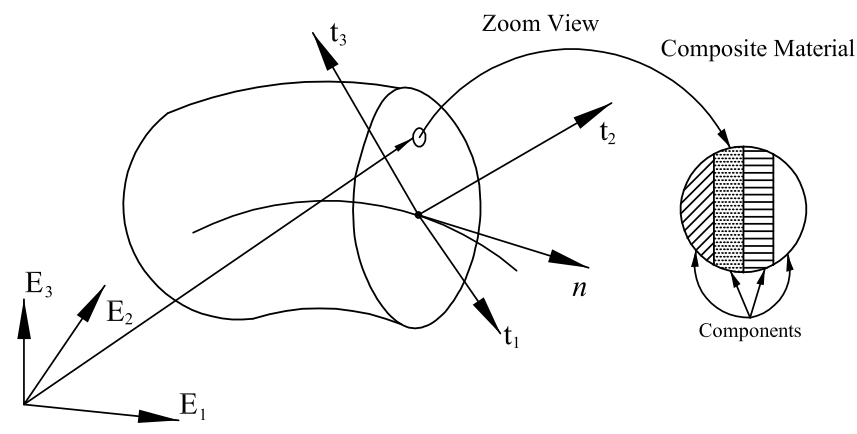

Fig. 2. Composite material associated to a material point on the crosssection. 
resulting composite is obtained using the mixing theory [9] which will be briefly described in following.

\subsection{Damage model}

Considering that the components of any spatial vector or tensor in the local frame $\left\{\hat{t}_{i}\right\}$ are the same as those of their corresponding material forms described in the inertial material frame $\left\{\widehat{E}_{i}\right\}$, the rate dependent damage model is formulated in terms of the material forms of the FPK stress vector $\widehat{P}_{1}^{\mathrm{m}}$ and, the strain and strain rate vectors, $\widehat{\mathscr{E}}_{n}$ and $\widehat{\mathscr{S}}_{n}$, respectively.

In Ref. [35] an isotropic rate independent damage model has been already presented. The model is able to differentiate the mechanical response for the tension or compression components of the stress vector [16]. Cracking is interpreted as a local effect depending on the evolution of the (scalar) internal damage variable denoted by $d \in[0,1]$, which measures the lack of secant stiffness of the material [36]. The variable $d$ is equal to zero before loading but when damage increases $d \rightarrow 1$, as it can be seen in Fig. 3 $[35,39]$.

Starting from an appropriated form of the free energy density for thermally stable problems considering the

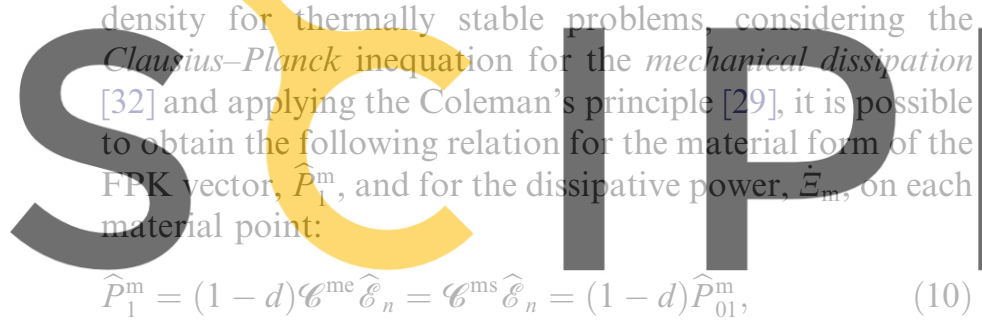

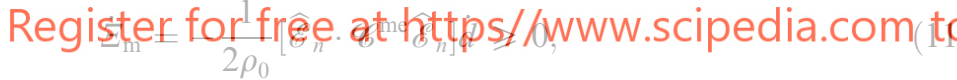

where $\rho_{0}$ is the mass density in the curved reference configuration and $\mathscr{C}^{\mathrm{me}}=\operatorname{diag}\left[E^{0}, G^{0}, G^{0}\right]$ is the material form of the elastic constitutive tensor, being $E^{0}$ and $G^{0}$ the axial and shear undamaged elastic modulus, $\mathscr{C}^{\mathrm{ms}}$ is the material secant constitutive tensor and $\widehat{P}_{01}^{\mathrm{m}}$ corresponds to the material descriptions of the elastic FPK stress vector. Eq. (10)

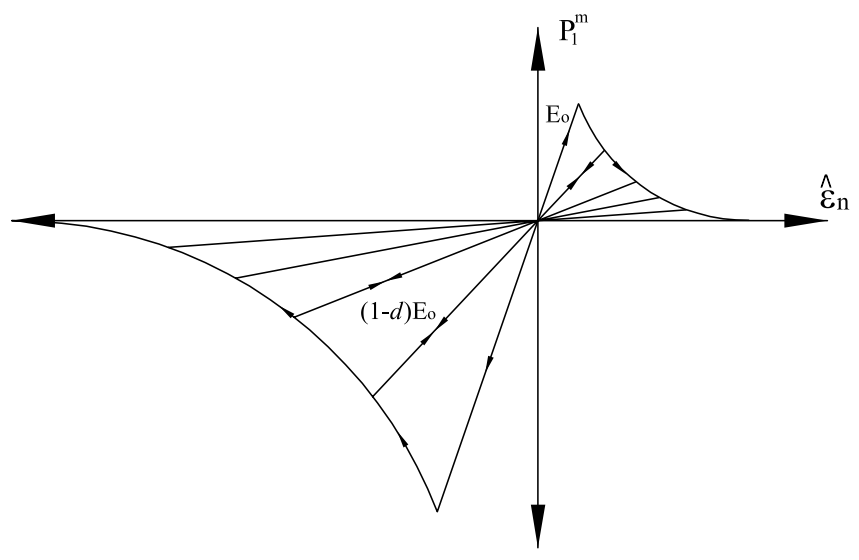

Fig. 3. Damage behavior at material point level. shows that the FPK stress vector is obtained from its elastic (undamaged) counterpart by multiplying it by the degrading factor $(1-d)$.

The damage yield criterion, $\mathscr{F}$, is written in terms of the material form of the undamaged principal stresses, $\widehat{P}_{p 0}^{\mathrm{m}}$, as $[7,17,38]$

$\mathscr{F}=\mathscr{P}^{\mathrm{m}}-f_{\mathrm{c}}=[1+r(n-1)] \sqrt{\sum_{i=1}^{3}\left(P_{p 0 i}^{\mathrm{m}}\right)^{2}}-f_{\mathrm{c}} \leqslant 0$,

where $\mathscr{P}^{\mathrm{m}}$ is the (scalar) equivalent stress. Additionally, the tension and compression strengths $f_{\mathrm{c}}$ and $f_{\mathrm{t}}$, the parts of the free energy developed when the tension or compression limits are reached, $\left(\Psi_{\mathrm{t}, \mathrm{c}}^{0}\right)_{L}$, and the parameters $r$ and $n$ are defined as
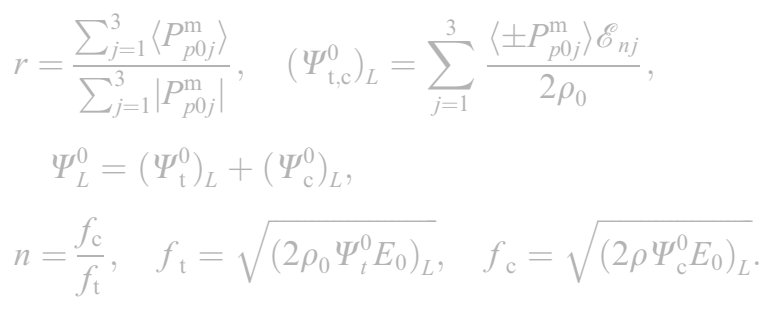

(13a)

The yield criterion used in Eq. (12) is based on measuring

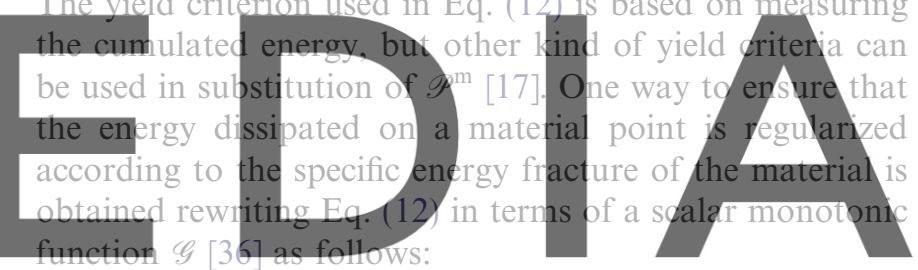
$\overline{\mathscr{F}}=\mathscr{G}\left(\mathscr{P}^{\mathrm{m}}\right)-\mathscr{G}\left(f_{c}\right)$

download the version without the watermark where $\mathscr{y}_{1}$ the new form of the yield criterion. The evolution law for the internal damage variable is given by $\dot{d}=\mu\left(\partial \overline{\mathscr{F}} / \partial \mathscr{P}^{\mathrm{m}}\right)$, with $\mu \geqslant 0$ being the damage consistency parameter. In Refs. $[16,17,35]$ it has been shown that, after several algebraic manipulations, it is possible to obtain that

$\dot{\mu}=\dot{\mathscr{P}}^{\mathrm{m}}=\dot{f}_{\mathrm{c}}=\frac{\partial \mathscr{P}^{\mathrm{m}}}{\partial \widehat{P}_{1}^{\mathrm{m}}} \cdot \dot{\widehat{P}}_{1}^{\mathrm{m}}=\frac{\partial \mathscr{P}^{\mathrm{m}}}{\partial \widehat{P}_{1}^{\mathrm{m}}} \cdot \mathscr{C}^{\mathrm{ms}} \dot{\widehat{\mathscr{E}}}_{n}$,

$\dot{d}=\frac{\mathrm{d} \mathscr{G}}{\mathrm{d} \mathscr{P}^{\mathrm{m}}} \dot{\mathscr{P}}^{\mathrm{m}}$,

$\dot{\Xi}_{\mathrm{m}}=\Psi_{0} \frac{\mathrm{d} \mathscr{G}}{\mathrm{d} \mathscr{P}^{\mathrm{m}}} \frac{\partial \mathscr{P}^{\mathrm{m}}}{\partial \widehat{P}_{1}^{\mathrm{m}}} \cdot \mathscr{C}^{\mathrm{ms}} \dot{\hat{\mathscr{E}}}_{n}$,

where $\Psi_{0}$ is the elastic (undamaged) free energy density. Finally, the Kuhn-Thucker relations have to be employed to derive the unloading-reloading conditions as it can be reviewed in [35]. Oliver et al. [36] propose an expression for $\mathscr{G}$ where it depends on a scalar parameter, which is calibrated to obtain an amount of dissipated energy equal to the specific fracture energy density of the material $g_{\mathrm{f}}$. The parameter $g_{\mathrm{f}}$ is derived from the fracture mechanics as $g_{\mathrm{f}}^{\mathrm{d}}=G_{\mathrm{f}}^{\mathrm{d}} / l_{\mathrm{c}}$, being $G_{\mathrm{f}}^{\mathrm{d}}$ the tensile fracture energy and $l_{\mathrm{c}}$ is the characteristic length of the fractured domain, that leads to the objective solution for the tensile and compressive cases $[9,36,39]$. 
Finally, after several algebraic manipulations [7,17], the material form of the tangent constitutive tensor $\mathscr{C}^{\mathrm{mt}}$ is calculated as

$$
\begin{aligned}
\delta \widehat{P}_{1}^{\mathrm{m}} & =\mathscr{C}^{\mathrm{mt}} \delta \widehat{\mathscr{E}}_{n} \\
& =\left[(1-d) \mathbf{I}-\frac{\mathrm{d} G}{\mathrm{~d} \mathscr{P}^{\mathrm{m}}} \widehat{P}_{01}^{\mathrm{m}} \otimes \frac{\partial \mathscr{P}^{\mathrm{m}}}{\partial \widehat{P}_{01}^{\mathrm{m}}}\right] \mathscr{C}^{\mathrm{m} e} \delta \widehat{\mathscr{E}}_{n},
\end{aligned}
$$

where $\mathbf{I}$ is the identity tensor and $\mathscr{C}^{\mathrm{mt}}$ is nonsymmetric and stress dependent.

\subsection{Viscosity}

The rate dependent behavior in each compounding material is considered by using the Maxwell's model [16]. In this case, the material form of the total FPK stress vector $\widehat{P}_{1}^{\mathrm{mt}}$ is obtained as the sum of the rate independent part $\widehat{P}_{1}^{\mathrm{m}}$, Eq. (10), and the viscous component $\widehat{P}_{1}^{\mathrm{mv}}$ as
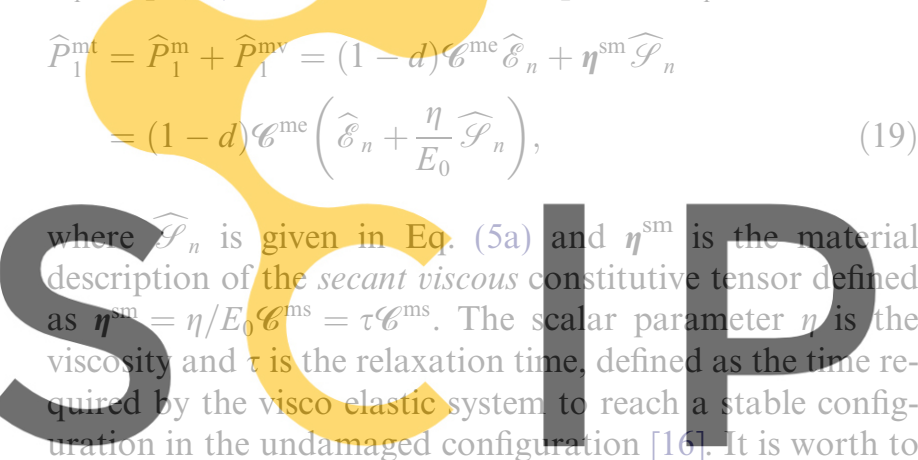
note that in Eq. (19), for the case of a material completely

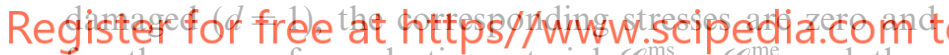
lor the case of an elastic material $\mathscr{C}^{\mathrm{ms}}=\mathscr{C}^{\mathrm{me}}$ and then the pure visco elastic behavior is recovered. In this case the dissipative power is given by

$\dot{\Xi}_{\mathrm{m}}=\left[\Psi_{0}+\frac{\tau}{\rho_{0}} \dot{\widehat{\mathscr{E}}}_{n} \cdot \mathscr{C}^{\mathrm{me}} \widehat{\mathscr{E}}\right] \frac{\mathrm{d} \mathscr{G}}{\mathrm{d} \mathscr{P}^{\mathrm{m}}} \frac{\partial \mathscr{P}^{\mathrm{m}}}{\partial \widehat{P}_{1}^{\mathrm{m}}} \cdot \mathscr{C}^{\mathrm{ms}} \dot{\widehat{\mathscr{E}}}_{n}$.

The linearized increment of the material form of the FPK stress vector is calculated as

$$
\begin{aligned}
\Delta \widehat{P}_{1}^{\mathrm{mt}} & =\mathscr{C}^{\mathrm{mt}} \Delta \widehat{\mathscr{E}}_{n}+\Delta \boldsymbol{\eta}^{\mathrm{sm}} \widehat{\mathscr{S}}_{n}+\boldsymbol{\eta}^{\mathrm{sm}} \Delta \widehat{\mathscr{S}}_{n} \\
& =\mathscr{C}^{\mathrm{mt}} \Delta \widehat{\mathscr{E}}_{n}-\Delta d \frac{\eta}{E_{0}} \mathscr{C}^{\mathrm{me}} \widehat{\mathscr{S}}_{n}+\boldsymbol{\eta}^{\mathrm{sm}} \Delta \widehat{\mathscr{S}}_{n} \\
& =\mathscr{C}^{\mathrm{mt}} \Delta \widehat{\mathscr{E}}_{n}-\Delta d \widehat{P}_{1}^{\mathrm{mv} 0}+\boldsymbol{\eta}^{\mathrm{sm}} \Delta \widehat{\mathscr{S}}_{n} \\
& =\mathscr{C}^{\mathrm{mv}} \Delta \widehat{\mathscr{E}}_{n}+\boldsymbol{\eta}^{\mathrm{sm}} \Delta \widehat{\mathscr{S}}_{n},
\end{aligned}
$$

where $\widehat{P}_{1}^{\mathrm{mv} 0}$ is the material description of the FPK visco elastic stress vector. An explicit expression for $\Delta \widehat{\mathscr{S}}_{n}$ in Eq. (21) is given in Appendix A and $\mathscr{C}^{\mathrm{mv}}$ is the material description of the tangent constitutive tensor which considers the viscous effect and is given by [7]

$$
\mathscr{C}^{\mathrm{mv}}=(1-d) \mathbf{I}+\frac{\mathrm{d} G}{\mathrm{~d} \mathscr{P}^{\mathrm{m}}}\left(\widehat{P}_{01}^{\mathrm{m}}+\widehat{P}_{1}^{\mathrm{mv} 0}\right) \otimes \frac{\partial \mathscr{P}^{\mathrm{m}}}{\partial \widehat{P}_{01}^{\mathrm{m}}} .
$$

The co-rotated version and the spatial description of the linearized increment of the FPK stress vector is obtained by the push-forward of Eq. (21) as

$$
\Delta\left[\widehat{P}_{1}^{\mathrm{t}}\right]=\mathscr{C}^{\mathrm{sv}} \Delta\left[\hat{\varepsilon}_{n}\right]+\boldsymbol{\eta}^{\mathrm{ss}} \Delta\left[\hat{\boldsymbol{s}}_{n}\right] ; \quad \Delta \widehat{P}_{1}^{\mathrm{t}}=\Delta\left[\widehat{P}_{1}^{\mathrm{t}}\right]+\Delta \tilde{\boldsymbol{\theta}} \widehat{P}_{1}^{\mathrm{t}}
$$

where $\mathscr{C}^{\mathrm{sv}}=\boldsymbol{\Lambda} \mathscr{C}^{\mathrm{mv}} \boldsymbol{\Lambda}^{\mathrm{T}}$ and $\boldsymbol{\eta}^{\mathrm{ss}}=\boldsymbol{\Lambda} \boldsymbol{\eta}^{\mathrm{sm}} \boldsymbol{\Lambda}^{\mathrm{T}}$ are the spatial descriptions of the rate dependent tangent and the secant viscous constitutive tensors, respectively; and $\Delta \tilde{\boldsymbol{\theta}}$ is the skew symmetric tensor associated to the linearized increment of the rotation vector, $\Delta \hat{\theta}$.

\subsection{Mixing theory for composites}

Each material point on the beam cross-section is treated as a composite material according to the mixing theory as described in Refs. $[35,39]$; therefore, in a generic material point coexists $N$ different components, all of them subjected to the same material strain $\widehat{\mathscr{E}}_{n}$, i.e. $\widehat{\mathscr{E}}_{n}=$ $\left(\widehat{\mathscr{E}}_{n}\right)^{1}=\cdots=\left(\widehat{\mathscr{E}}_{n}\right)^{j}=\cdots=\left(\widehat{\mathscr{E}}_{n}\right)^{N}$, forcing the strain compatibility between components. The free energy density and the dissipation of the composite is obtained as the weighted sum of the free energy densities and the dissipation of the components, respectively [39]. The weighting

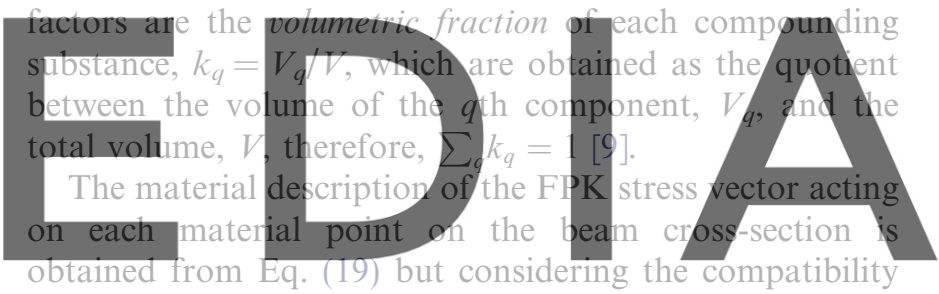
condition, which leads to the material form of the secant

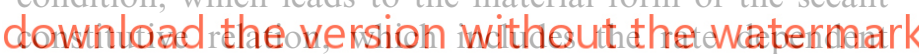
effects and is given by

$$
\begin{aligned}
\widehat{P}_{1}^{\mathrm{mt}} & \equiv \sum_{q}^{N} k_{q}\left(\widehat{P}_{1}^{\mathrm{m}}+\widehat{P}_{1}^{\mathrm{mv}}\right)_{q} \\
& =\sum_{q}^{N} k_{q}\left[(1-d) \mathscr{C}^{\mathrm{me}}\left(\widehat{\mathscr{E}}_{n}+\frac{\eta}{E_{0}} \widehat{\mathscr{S}}_{n}\right)\right]_{q}
\end{aligned}
$$

Stress resultant and couples are then calculated employing Eq. (6). The mixing rule provides an appropriated framework for simulating the mechanical behavior of some advanced composed materials such as: epoxy based materials with glass or carbon fibers or even reinforced concrete [39]. Due to the limitations imposed by the planarity of the cross-section, the incorporation of transversal reinforcement is not allowed in the present formulation. However, the simulation of their effects can be carried out by means of modifying the fracture energy and the yielding point of the materials.

The co-rotated form of the linearized relation between strains and stresses for the composite material is based on the weighted sum of the spatial form of the tangent constitutive tensors $\left(\mathscr{C}^{\mathrm{sv}}\right)_{q}$ plus the rate dependent tensors $\left(\boldsymbol{\eta}^{\mathrm{ss}}\right)_{q}$ of Eq. (22) for each one of the components $q$ and it is given by 


$$
\begin{aligned}
\Delta\left[\widehat{P}_{1}^{\mathrm{t}}\right] & =\sum_{q=1}^{N} k_{q}\left(\mathscr{C}^{\mathrm{sv}}\right)_{q} \Delta\left[\hat{\varepsilon}_{n}\right]+\sum_{q=1}^{N} k_{q}\left(\boldsymbol{\eta}^{\mathrm{ss}}\right)_{q} \Delta\left[\hat{s}_{n}\right] \\
& =\overline{\mathscr{C}}^{\mathrm{sv}} \Delta\left[\hat{\varepsilon}_{n}\right]+\overline{\boldsymbol{\eta}}^{\mathrm{ss}} \Delta\left[\hat{\boldsymbol{s}}_{n}\right] .
\end{aligned}
$$

In the same manner, the spatial descriptions of the linearized FPK stress vector is obtained as

$\Delta \widehat{P}_{1}^{\mathrm{t}}=\Delta\left[\widehat{P}_{1}^{\mathrm{t}}\right]+\Delta \tilde{\boldsymbol{\theta}} \widehat{P}_{1}^{\mathrm{t}}=\overline{\mathscr{C}}^{\mathrm{sv}} \Delta\left[\hat{\varepsilon}_{n}\right]+\overline{\boldsymbol{\eta}}^{\mathrm{ss}} \Delta\left[\hat{\boldsymbol{s}}_{n}\right]+\Delta \tilde{\boldsymbol{\theta}} \widehat{P}_{1}^{\mathrm{t}}$.

The linearized form of $\hat{n}$ and $\hat{m}$ is obtained starting from Eq. (26) and integrating over the cross-section as

$$
\left[\begin{array}{c}
\Delta \hat{n} \\
\Delta \hat{m}
\end{array}\right]=\underbrace{\left[\begin{array}{ll}
\overline{\mathbf{C}}_{11}^{\mathrm{sv}} & \overline{\mathbf{C}}_{12}^{\mathrm{sv}} \\
\overline{\mathbf{C}}_{21}^{\mathrm{sv}} & \overline{\mathbf{C}}_{22}^{\mathrm{sv}}
\end{array}\right]}_{\left[\overline{\mathbf{C}}^{\mathrm{sv}}\right]}\left[\begin{array}{c}
\Delta\left[\hat{\gamma}_{n}^{\nabla}\right] \\
\Delta\left[\hat{\omega}_{n}^{\nabla}\right]
\end{array}\right]
$$
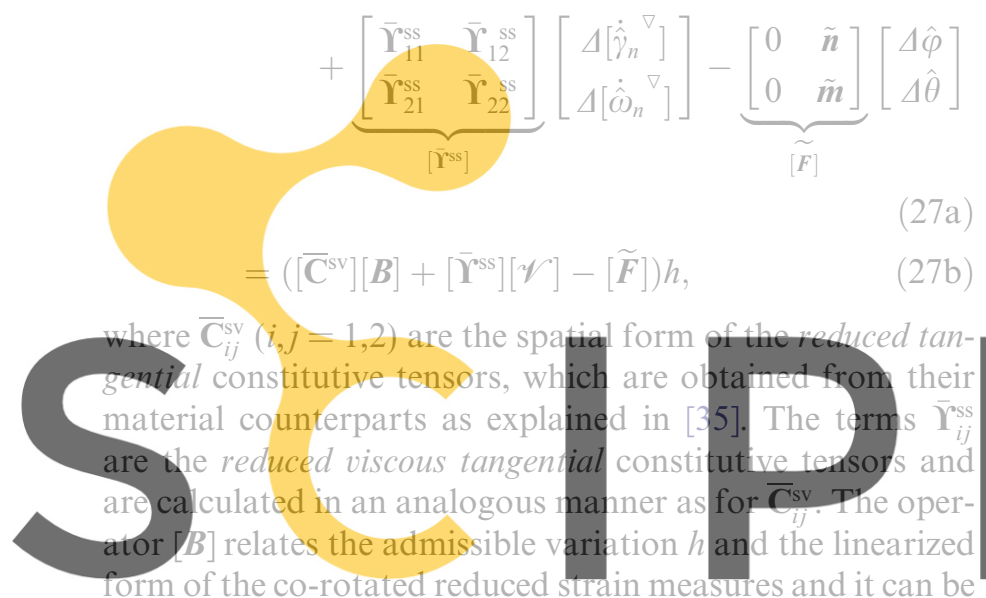

found in $[48]$ and $[y \mathcal{C}]$ relates the linearized form of the co-

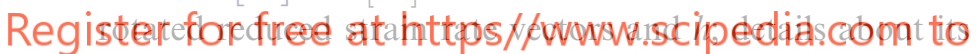
deduction are given in Appendix A. It is worth to note that in the present formulation both $[\overline{\mathbb{C}}$ sv $]$ and $\left[\overline{\mathfrak{Y}}^{\text {ss }}\right]$ are rate and configuration dependent operators.

\section{Numerical implementation}

The Newton type numerical solution procedure is normally constructed starting from the linearization of the weak form of Eq. (9) $[19,48]$. The nonlinear functional $\mathbf{G}(\hat{\varphi}, \boldsymbol{\Lambda}, h)$ can be expressed as the sum of three terms corresponding to the contribution of internal, external and inertial terms, $\mathbf{G}_{\text {int }}, \mathbf{G}_{\text {ext }}, \mathbf{G}_{\text {ine }}$, respectively, then its linearization can be expressed as

$$
\begin{aligned}
& \mathscr{L}\left[\mathbf{G}\left(\hat{\varphi}_{*}, \boldsymbol{\Lambda}_{*}, h, p\right)\right]=\underbrace{\mathbf{G}_{\text {int }}+\mathbf{G}_{\text {ext }}+\mathbf{G}_{\text {ine }}}_{\mathbf{G}\left(\hat{\varphi}_{*}, \boldsymbol{\Lambda}_{*}, h\right)} \\
& +\underbrace{\left[D \mathbf{G}_{\text {int }}+D \mathbf{G}_{\text {ext }}+D \mathbf{G}_{\text {ine }}\right] \cdot p}_{D \mathbf{G}\left(\hat{\varphi}_{*}, \boldsymbol{\Lambda}_{*}, h\right) \cdot p},
\end{aligned}
$$

where $\mathscr{L}\left[\mathbf{G}\left(\hat{\varphi}_{*}, \boldsymbol{\Lambda}_{*}, h, p\right)\right]$ is the linear part of the functional $\mathbf{G}(\hat{\varphi}, \boldsymbol{\Lambda}, h)$ at the configuration $(\hat{\varphi}, \boldsymbol{\Lambda})=\left(\hat{\varphi}_{*}, \boldsymbol{\Lambda}_{*}\right)$ and $p \approx$ $(\Delta \hat{\varphi}, \Delta \hat{\theta}) \in \mathbb{R}^{3} \times T_{\Lambda}^{\text {spa }}$ denotes an admissible variation. The term $\mathbf{G}\left(\hat{\varphi}_{*}, \boldsymbol{\Lambda}_{*}, h\right)$ supplies the unbalanced force term and $D \mathbf{G}\left(\hat{\varphi}_{*}, \boldsymbol{\Lambda}_{*}, h\right) \cdot p$ the tangential stiffness $[19,48]$.
Some branches of engineering such as robotics, the study of flexible mechanisms and some approaches in earthquake engineering are focused on the dynamic response of damped system but considering that the material behavior remains elastic. In some cases, several 'ad hoc' approximations have been developed, most of them based on adding a damping term to the equilibrium equations, which is considered to be a linear function of the strain rates $[20,23]$. The approach proposed in this work makes hypothesis only at constitutive level which implies that the structural damping is added to the system in the term $\mathbf{G}_{\text {int }}\left(\hat{\varphi}_{*}, \boldsymbol{\Lambda}_{*}, h\right)$ of Eq. (28) by means of the stress resultant and couples, Eq. (6).

\subsection{Tangential stiffness}

The linearization of the internal and external terms and its numerical implementation has been already published in $[19,48]$ for the case of linear elastic materials. For the case of an arbitrary distribution of materials with nonlinear rate independent constitutive equations, the results can be reviewed in Refs. $[35,41]$. However, if rate dependent constitutive laws are employed, the linearization of $\mathrm{G}_{\text {int }}$ pro-

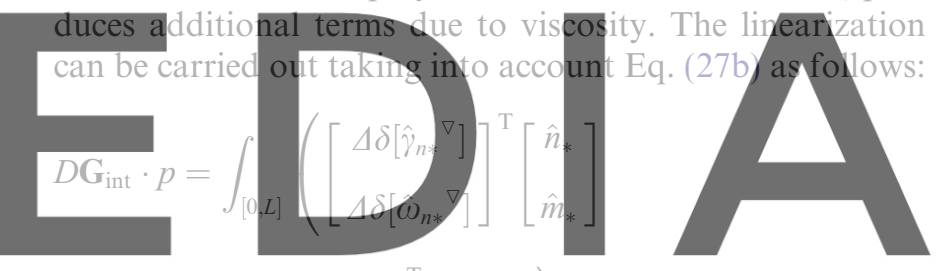
download thegryersion waithout the watermark

$$
\begin{gathered}
=\underbrace{\int_{[0, L]} h^{\mathrm{T}}\left[\boldsymbol{B}_{*}\right]^{\mathrm{T}}\left[\overline{\mathbf{C}}_{*}^{\mathrm{sV}}\right]\left[\boldsymbol{B}_{*}\right] p \mathrm{~d} S}_{\mathbf{K}_{\mathrm{M}}} \\
+\underbrace{\int_{[0, L]} h^{\mathrm{T}}\left(\left[\tilde{\boldsymbol{n}}_{*, S}\right]-\left[\widetilde{\boldsymbol{F}}_{*}\right]\right) p \mathrm{~d} S_{\mathbf{K}_{\mathrm{G}}}}_{\mathbf{K}_{\mathrm{V}}} \\
+\underbrace{\int_{[\mathrm{T}} h^{\mathrm{T}}\left[\mathbf{B}_{*}\right]^{\mathrm{T}}\left[\overline{\mathbf{Y}}_{*}^{\mathrm{st}}\right]\left[\mathscr{V}_{*}\right] p \mathrm{~d} S}_{[0, L]},
\end{gathered}
$$

where the subscript $*$ indicates that the involved quantities have to be evaluated at $(\hat{\varphi}, \boldsymbol{\Lambda})=\left(\hat{\varphi}_{*}, \boldsymbol{\Lambda}_{*}\right)$. The explicit form of the stress dependent operator $\left[\tilde{\boldsymbol{n}}_{*, S}\right]$ can be consulted in [35], $\mathbf{K}_{G}, \mathbf{K}_{M}$ give the geometric and the constitutive parts of the tangential stiffness. The additional term $\mathbf{K}_{\mathrm{V}}$ gives the viscous part, which considers the rate dependent contribution of the constitutive laws. By the other hand, the linearization of the term $\mathbf{G}_{\text {int }}$ can be carried out phrasing its spatial form in terms of the material quantities: $\mathbf{I}_{\rho 0}=\Lambda^{\mathrm{T}} \mathscr{I}_{\rho 0} \boldsymbol{\Lambda}, \widehat{A}_{n}=\boldsymbol{\Lambda}^{\mathrm{T}} \hat{\alpha}_{n}$ and $\widehat{V}_{n}=\boldsymbol{\Lambda}^{\mathrm{T}} \hat{v}_{n}$, and taking into account the push-forward relations, in the following form: 


$$
\begin{aligned}
D \mathbf{G}_{\text {ine }} \cdot h & =\int_{[0, L]} h^{\mathrm{T}} \Delta\left[\begin{array}{c}
\mathscr{A}_{\rho_{0}} \ddot{\hat{\varphi}} \\
\mathbf{\Lambda}\left\{\mathbf{I}_{\rho_{0}} \widehat{A}_{n}+\widetilde{\mathbf{V}}_{n}\left(\mathbf{I}_{\rho_{0}} \widehat{V}_{n}\right)\right\}
\end{array}\right] \mathrm{d} S \\
= & \int_{[0, L]} h^{\mathrm{T}}[\underbrace{\Delta \boldsymbol{\Lambda}\left\{\mathbf{I}_{\rho_{0}} \widehat{A}_{\rho_{0}} \Delta \ddot{\hat{\varphi}} \widetilde{\mathbf{V}}_{n}\left(\mathbf{I}_{\rho_{0}} \widehat{V}_{n}\right)\right\}}_{\mathrm{In}_{1}} \\
& +\underbrace{\Lambda\left(\Delta\left\{\mathbf{I}_{\rho_{0}} \widehat{A}_{n}+\widetilde{V}_{n}\left(\mathbf{I}_{\rho_{0}} \widehat{V}_{n}\right)\right\}\right)}_{\text {In }_{2}}] \mathrm{d} S .
\end{aligned}
$$

Using the notation $[\widetilde{\bullet}=\mathbf{\Pi}[\bullet]$ for the skew-symmetric operator, and $\ddot{\ddot{\theta}}$ considering that $\Delta \widehat{V}_{n}=\boldsymbol{\Lambda}^{\mathrm{T}}$ $\Delta \hat{\theta}$ and $\Delta \widehat{A}_{n}=\boldsymbol{\Lambda}^{\mathrm{T}} \Delta \hat{\theta}-\widehat{V}_{n} \times \boldsymbol{\Lambda}^{\mathrm{T}} \Delta \hat{\theta}[10]$, it is possible to write the terms $\operatorname{In}_{1}$ and $\operatorname{In}_{2}$ of Eq. (30) as

$\operatorname{In}_{1}=-\Pi\left[\mathscr{I}_{\rho_{0}} \hat{\alpha}_{n}+\tilde{v}_{n} \mathscr{I}_{\rho_{0}} \hat{v}_{n}\right] \Delta \hat{\theta}$

(31a)

$\operatorname{In}_{2}=\mathscr{I}_{\rho_{0}} \Delta \ddot{\hat{\theta}}+\left\{\tilde{v}_{n} \mathscr{I} \rho_{0}-\mathscr{I}_{\rho_{0}} \tilde{\boldsymbol{v}}_{n}-\Pi\left[\mathscr{I}_{\rho_{0}} \hat{v}_{n}\right]\right\} \Delta \dot{\hat{\theta}}$

(31b)

Replacing the results of Eqs. (31a) and (31b) into Eq. (30) it is possible to rewrite the linearized form of the spatial description of the inertial term as

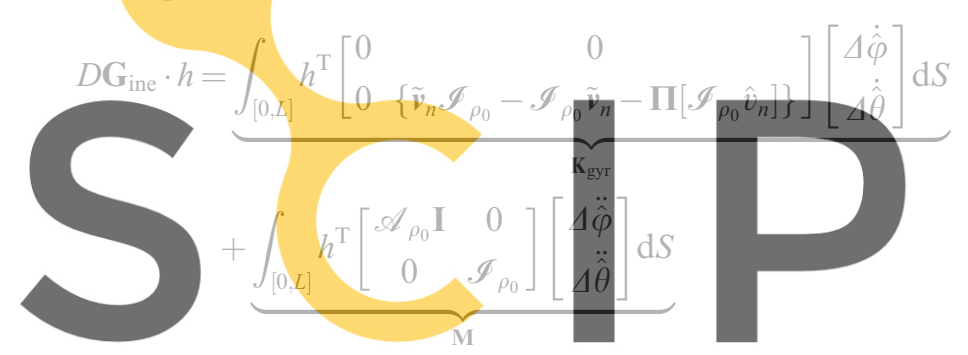

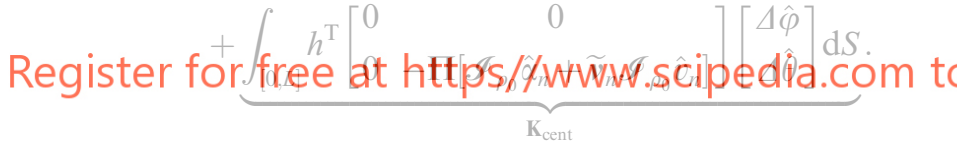

The terms $\mathbf{M}, \mathbf{K}_{\mathrm{gyr}}$ and $\mathbf{K}_{\text {cent }}$ of Eq. (32) correspond to the mass, gyroscopic and centrifugal tangential stiffness of the linearized problem [11]. The resulting inertial contribution to the tangential stiffness, $\left(\mathbf{M}+\mathbf{K}_{\mathrm{gyr}}+\mathbf{K}_{\text {cent }}\right)$, is nonsymmetric and configuration dependent on the rotational degrees of freedom, as it can be verified from the gyroscopic and centrifugal terms. Finally, Eq. (28) can be rewritten as

$\mathscr{L}[\mathbf{G}]=\mathbf{G}+\mathbf{K}_{M}+\mathbf{K}_{G}+\mathbf{K}_{V}+\mathbf{K}_{\mathrm{P}}+\mathbf{M}+\mathbf{C}_{\mathrm{gyr}}+\mathbf{K}_{\mathrm{cent}}$

where $\mathbf{K}_{\mathrm{P}}$ corresponds to the part of the tangent stiffness which is dependent on the loading and it can be consulted in $[48,49]$. The numerical implementation in a finite element (FE) code of the discrete form of Eq. (32) follows standard procedures and can be reviewed in Refs. $[19,31,48]$.

\subsection{Time steeping scheme and updating procedure}

In this section, the subscript $m$ denotes the discrete approximation of a varying quantity at time $t_{m}$. The basic
Table 1

Rotational part of Newmark's algorithm

$\widehat{\Psi}_{\mathrm{m}}=\Delta t\left(\widehat{V}_{\mathrm{m}}+\Delta t\left[\left(\frac{1}{2}-\beta\right) \widehat{A}_{\mathrm{m}}+\beta \widehat{A}_{m+1}\right]\right)$

$\widehat{V}_{m+1}=\widehat{V}_{\mathrm{m}}+\Delta t\left[(1-\gamma) \widehat{A}_{\mathrm{m}}+\gamma \widehat{A}_{m+1}\right]$

problem is: starting from a configuration $\left(\hat{\varphi}_{m}, \boldsymbol{\Lambda}_{\mathrm{m}}\right) \in \mathbb{R}^{3} \times S O(3)$, its associated linear and angular velocities and accelerations, written in spatial description by convenience, $\left(\dot{\hat{\varphi}}_{m}, \ddot{\hat{\varphi}}_{m}, \hat{v}_{\mathrm{m}}, \hat{\alpha}_{\mathrm{m}}\right) \in \mathbb{R}^{3} \times T_{\boldsymbol{\Lambda}_{n}}^{\text {spa }}$, the updated configuration and the associated updated linear and angular velocities and accelerations at time $t_{m+1}$ has to be obtained consistently with the weak form of the balance equations.

Newmark's implicit time stepping algorithm has been chosen as integrating method following the development originally proposed by Simo et al. [49]. The algorithm for the configuration update can be written in either material or spatial description. For the translational part the timestepping procedure takes place in $\mathbb{R}^{3}$ and the configuration is updated employing the classical version of the algorithm for elastodynamics. For the rotational part, the time-stepping procedure takes place in $S O(3)$ and the basic steps are

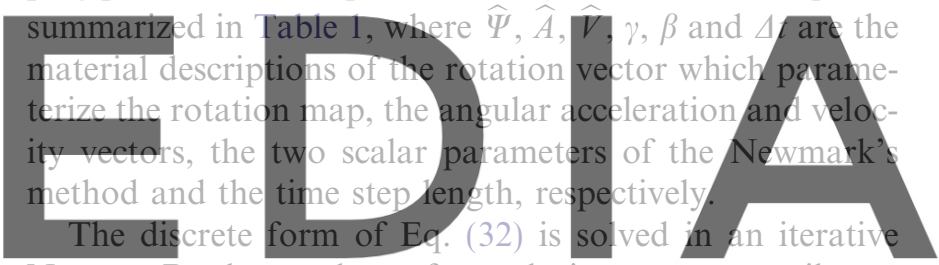

Newton-Raphson scheme for each time step $t_{m}$, until convergence is achieved. Each iteration in a time step is

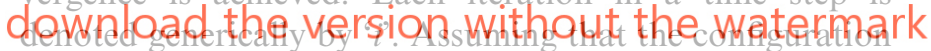
in each node of the FE mesh $\left(\hat{\varphi}_{\mathrm{m}}^{(i)}, \Lambda_{\mathrm{m}}^{(i)}\right)$ is known and solving the linearized system, an incremental field $\left(\Delta \hat{\varphi}_{\mathrm{m}}^{(i)}, \Delta \hat{\theta}_{\mathrm{m}}^{(i)}\right)$ is obtained; where $\Delta \hat{\theta}_{\mathrm{m}}^{(i)}$ is the iterative rotation vector, which parameterize the iterative rotation tensor [19]. The rotational component of the configuration is updated using the exponential map $(\exp [\bullet])$ as $[48]$

$\boldsymbol{\Lambda}_{m+1}^{(i+1)}=\exp \left[\Delta \widetilde{\boldsymbol{\Theta}}_{\mathrm{m}}^{(i)}\right] \boldsymbol{\Lambda}_{m+1}^{(i)}=\exp \left[\widetilde{\boldsymbol{\Theta}}_{\mathrm{m}}^{(i+1)}\right] \boldsymbol{\Lambda}_{\mathrm{m}}$,

where $\Delta \widetilde{\boldsymbol{\Theta}}_{\mathrm{m}}^{(i)}$ and $\widetilde{\boldsymbol{\Theta}}_{\mathrm{m}}^{(i+1)}$ are the skew-symmetric tensors associated to the iterative and incremental rotation vectors which parameterizes the iterative and incremental rotation tensors from $\boldsymbol{\Lambda}_{m+1}^{(i)}$ and $\boldsymbol{\Lambda}_{m}$ to $\boldsymbol{\Lambda}_{m+1}^{(i+1)}$, respectively. Note that $\Delta \hat{\theta}_{\mathrm{m}}^{(i)}$ is non-additive to $\hat{\theta}_{\mathrm{m}}^{(i)}$, but they are related by

$\hat{\theta}_{\mathrm{m}}^{(i+1)}=\mathbf{T}\left(\hat{\theta}_{\mathrm{m}}^{(i)}\right) \Delta \hat{\theta}_{\mathrm{m}}^{(i)}+\hat{\theta}_{\mathrm{m}}^{(i)}$

where the explicit form of the tensor $\mathbf{T}$ can be consulted in $[33,48]$.

The updating procedure for angular velocity $\widehat{V}_{m+1}^{(i+1)}$ and acceleration $\widehat{A}_{m+1}^{(i+1)}$ is carried out in material form because this description is advantageous to write time-stepping algorithms in $S O(3)[26,49]$. After some algebraic manipulations and considering that $\widehat{\Psi}_{\mathrm{m}}^{(i)}=\boldsymbol{\Lambda}_{\mathrm{m}}^{\mathrm{T}} \hat{\theta}_{\mathrm{m}}^{(i)}$ and $\widehat{\Psi}_{\mathrm{m}}^{(i+1)}=$ $\boldsymbol{\Lambda}_{\mathrm{m}}^{\mathrm{T}} \hat{\theta}_{\mathrm{m}}^{(i+1)}$, we obtain the updated angular acceleration and velocity vectors in material description as follows: 
$\widehat{A}_{m+1}^{(i+1)}=\widehat{A}_{m+1}^{(i)}+\frac{1}{(\Delta t)^{2} \beta}\left[\widehat{\Psi}_{\mathrm{m}}^{(i+1)}-\widehat{\Psi}_{\mathrm{m}}^{(i)}\right]$

$\widehat{V}_{m+1}^{(i+1)}=\widehat{V}_{m+1}^{(i)}+\frac{\gamma}{\Delta t \beta}\left[\widehat{\Psi}_{\mathrm{m}}^{(i+1)}-\widehat{\Psi}_{\mathrm{m}}^{(i)}\right]$

Their spatial counterparts are obtained through the pushforward relations $\hat{v}_{m+1}^{(i+1)}=\boldsymbol{\Lambda}_{m+1}^{(i+1)} \widehat{\Omega}_{m+1}^{(i+1)}$, and $\hat{\alpha}_{m+1}^{(i+1)}=$ $\boldsymbol{\Lambda}_{m+1}^{(i+1)} \widehat{A}_{m+1}^{(i+1)}$. This updating procedure applies for $i \geqslant 1$. For $i=0$, the 'initial guess' in the Newton process, one sets $\boldsymbol{\Lambda}_{m+1}^{(0)}=\boldsymbol{\Lambda}_{\mathrm{m}}$ with $\hat{v}_{m+1}^{(0)}$ and $\hat{\alpha}_{m+1}^{(0)}$ computed with the formulae given in Table 1.

\subsection{Iterative procedure for updating the strain and strain rate vectors}

Normally, in the nonlinear FEM approach it is necessary to estimate the strain and strain rate measures at integration point level. Assuming that for a given time step $(m)$ and iteration (i) the non converged configuration $\left(\hat{\varphi}_{\mathrm{m}}^{(i)}, \boldsymbol{\Lambda}_{\mathrm{m}}^{(i)}\right)$ and the associated dynamic variables $\dot{\hat{\varphi}}_{\mathrm{m}}, \ddot{\hat{\varphi}}_{\mathrm{m}}, \hat{v}_{\mathrm{m}}^{(i)}, \hat{\alpha}_{\mathrm{m}}^{(i)}$ are known, it is possible to estimate the spatial description of the translational and rotational reduced strain vectors, relative to the curved reference beam, as

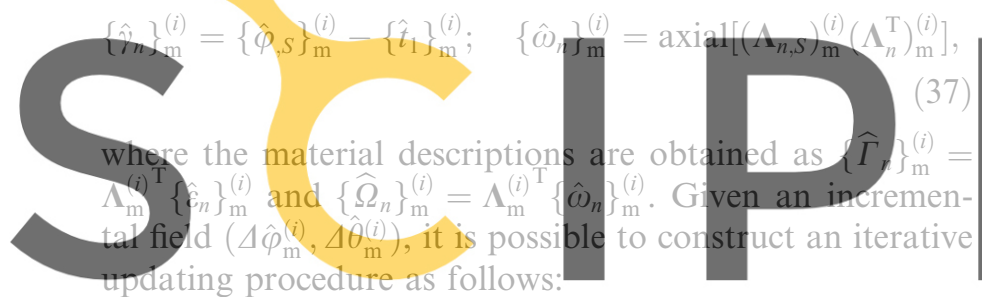

$$
\begin{aligned}
\left\{s_{n}\right\}_{\mathrm{m}}^{(i+1)}= & \left\{\hat{v}_{, S}\right\}_{\mathrm{m}}^{(i+1)}-\left\{\tilde{\boldsymbol{v}}_{n}\right\}_{\mathrm{m}}^{(i+1)}\left\{\hat{\varphi}_{, S}\right\}_{\mathrm{m}}^{(i+1)} \\
& +\left\{\tilde{\boldsymbol{v}}_{n, S}\right\}_{\mathrm{m}}^{(i+1)}\left(\xi_{\beta} \widehat{E}_{\beta}\right) .
\end{aligned}
$$

The material counterpart of Eq. (40) is obtained by means of the pullback by $\boldsymbol{\Lambda}_{\mathrm{m}}^{(i+1)}$. In Eq. (40) it has been supposed that the nodal values of the angular velocity tensors are interpolated at the integration points by means of using an isoparametric approximation, i.e. $\quad\left\{\tilde{\boldsymbol{v}}_{n}\right\}_{\mathrm{m}}^{(i+1)}(S)=$ $\sum_{I}^{N_{p}} N_{I}(S)\left\{\tilde{\boldsymbol{v}}_{n}^{I}\right\}_{\mathrm{m}}^{(i+1)}$ as usual in the FE method and, therefore, the term $\left\{\tilde{\boldsymbol{v}}_{, S}\right\}_{\mathrm{m}}^{(i+1)}$ is calculated as $\sum_{I}^{N_{p}} N_{I}(S)_{S}$ $\left\{\tilde{\boldsymbol{v}}_{n}^{\mathrm{I}}\right\}_{\mathrm{m}}^{(i+1)}$, where $N_{p}$ is the number of nodal points on a beam element.

\subsection{Cross-sectional analysis}

As it has been explained in Section 3, the distribution of materials on the beam cross-sections can be arbitrary. From the point of view of the numerical implementation, the cross-section is meshed into a grid of quadrilaterals, each of them corresponding to a fiber oriented along the beam axis, as it can be seen in Fig. 4. The geometry of each quadrilateral is described by means of normalized bidimensional shapes functions as usual in the FE method.

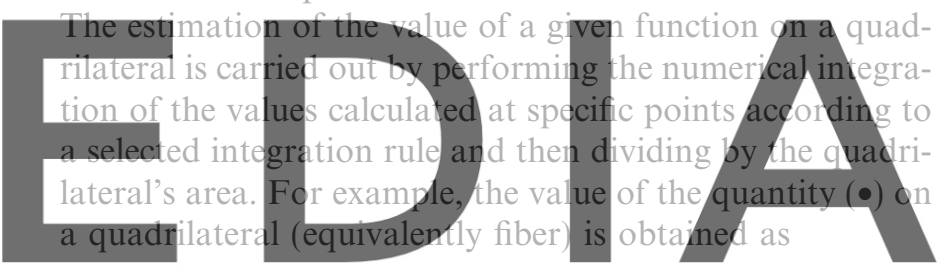

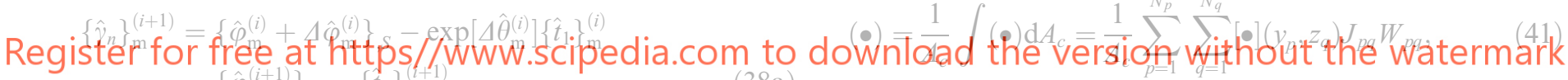

$\begin{aligned} & =\left\{\varphi_{\mathrm{m}}\right\}_{, S}-\left\{t_{1}\right\}_{\mathrm{m}}, \\ \left\{\hat{\omega}_{n}\right\}_{\mathrm{m}}^{(i+1)} & =\operatorname{axial}\left[\Delta\left\{\tilde{\omega}_{n}\right\}_{\mathrm{m}}^{(i)}+\exp \left[\Delta \hat{\theta}_{\mathrm{m}}^{(i)}\right]\left\{\tilde{\omega}_{n}\right\}_{\mathrm{m}}^{(i)} \exp \left[\Delta \hat{\theta}_{\mathrm{m}}^{(i)}\right]^{\mathrm{T}}\right],\end{aligned}$

$\Delta\left\{\tilde{\boldsymbol{\omega}}_{n}\right\}_{\mathrm{m}}^{(i)}=\frac{\mathrm{d}\left(\exp \left[\Delta \hat{\theta}_{\mathrm{m}}^{(i)}\right]\right)}{\mathrm{d} S} \exp \left[-\Delta \hat{\theta}_{\mathrm{m}}^{(i)}\right]$

It is worth to note that the normal vector $\left\{\hat{t}_{1}\right\}_{\mathrm{m}}^{(i+1)}$ is obtained by means of applying the iterative rotation tensor $\exp \left[\Delta \hat{\theta}_{\mathrm{m}}^{(i)}\right]$. The explicit expression for the iterative (additive) curvature increment of Eq. (38c) can be consulted in [48]. The corresponding material description of Eqs. (38a) and (38b), $\left\{\widehat{\Gamma}_{n}\right\}_{\mathrm{m}}^{(i+1)}$ and $\left\{\widehat{\Omega}_{n}\right\}_{\mathrm{m}}^{(i+1)}$, can be obtained by means of the pullback by $\Lambda_{\mathrm{m}}^{(i+1)}$ and the material description of the strain vector of Eq. (3) is calculated according to

$\left\{\widehat{\mathscr{E}}_{n}\right\}_{\mathrm{m}}^{(i+1)}=\frac{1}{\left|\mathbf{F}_{0}\right|}\left\{\Gamma_{n}\right\}_{\mathrm{m}}^{(i+1)}+\left\{\widetilde{\boldsymbol{\Omega}}_{n}\right\}_{\mathrm{m}}^{(i+1)}\left(\xi_{2} \widehat{E}_{2}+\xi_{3} \widehat{E}_{3}\right)$

with $\left|\mathbf{F}_{0}\right|_{\mathrm{m}}^{(i+1)}=\left(\boldsymbol{\Lambda}_{0}^{\mathrm{T}} \hat{\varphi}_{0, S} \cdot \widehat{E}_{1}+\xi_{3} \widehat{\Omega}_{02}-\xi_{2} \widehat{\Omega}_{03}\right)$ [30], which is an initial geometric parameter.

Having estimated $\hat{\varphi}_{\mathrm{m}}^{(i+1)}, \dot{\hat{\varphi}}_{\mathrm{m}}^{(i+1)}$ and $\hat{v}_{\mathrm{m}}^{(i+1)}$ from Newmark's algorithm, it is possible to construct the discrete form of the co-rotated strain rate vector existing on each material point of the cross-section of the current beam relative to the curved reference beam, Eq. (5b), as where $A_{\mathrm{c}}$ is the area of the quadrilateral, $N_{p}$ and $N_{q}$ are the number of integration points in the two directions of the normalized geometry of the quadrilateral, $[\bullet]\left(y_{p}, z_{q}\right)$ is the value of $(\bullet)$ existing on an integration point with coordinates $\left(y_{p}, z_{q}\right)$ with respect to the reference beam axis, $J_{p q}$ is the Jacobian of the transformation between normalized coordinates and cross-sectional coordinates and $W_{p q}$ are the weighting factors.

In the numerical simulations, Eq. (41) is used to estimate the value of the components of the FPK stress vector and the coefficients of the tangent constitutive tensors. Having obtained the values of the FPK stress vector on each quadrilateral, the cross-sectional forces and moments are obtained as

$\hat{n}^{\mathrm{m}}=\sum_{k=1}^{N_{\text {fiber }}}\left(A_{c}\right)_{k}\left(\widehat{P}_{1}^{\mathrm{m}}\right)_{k}, \quad \hat{m}^{\mathrm{m}}=\sum_{k=1}^{N_{\text {fiber }}}\left(A_{c}\right)_{k} \hat{\ell}_{k} \times\left(\widehat{P}_{1}^{\mathrm{m}}\right)_{k}$,

where $N_{\text {fiber }}$ is the number of quadrilaterals of the crosssection, $\left(A_{\mathrm{c}}\right)_{k}$ and $\left(\widehat{P}_{1}^{\mathrm{m}}\right)_{k}$ are the area and average value of the FPK stress vector of the $k$ quadrilateral, and $\hat{\ell}_{k}=\left(0, y_{k}, z_{k}\right)$ are the coordinates of the gravity center of the $k$ th quadrilateral with respect to the beam axis. Eq. (42) correspond to the discrete form of Eq. (6). Having 


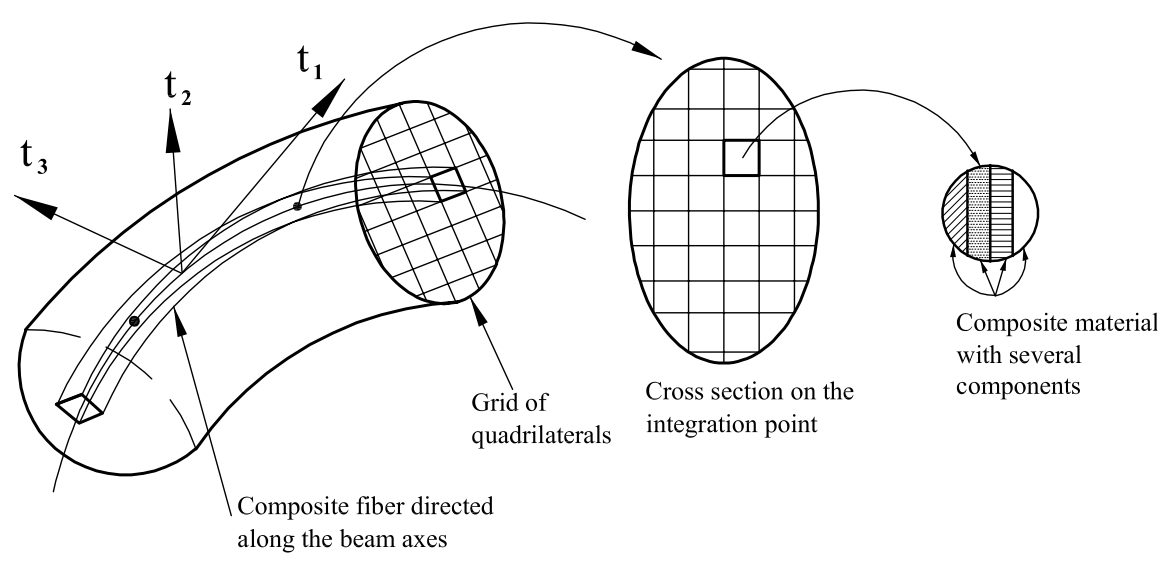

Fig. 4. Discrete fiber model of a beam element.

obtained the values of the tangent constitutive tensors, $\overline{\boldsymbol{C}}^{\mathrm{sv}}$ and $\overline{\boldsymbol{\eta}}^{\mathrm{ss}}$, at each fiber, the reduced constitutive tensor $\left[\overline{\mathbf{C}}^{\text {sv }}\right]$ is obtained as explained in Ref. [35] and $\left[\overline{\mathbf{Y}}^{\text {ss }}\right]$ is obtained analogously. Therefore, following the present approach, an additional integration loop running over the fibers is required to obtain the reduced quantities appearing in the beam theory. Additionally, a second loop is required to take into account the different simple materials of the composite associated to each fiber. In this stage the mixing theory is used to compute the stress vector and the tangent constitutive tensors.

The basic steps of the cross-sectional analysis carried out in each time step and iteration of the global NewtonRaphson scheme are summarized as: (i) Having obtained the reduced strain vectors, $\widehat{\Gamma}_{n}$ and $\widehat{\Omega}_{n}$, the material strain and strain rate vectors are calculated according to Eqs. (3) and (5a) and they are imposed for each simple material associated to the composite of a given fiber. (ii) At fiber level, the mixing theory and Eq. (41) are used to obtain the average stress level and tangent tensors. (iii) The integration procedure carried out over the fibers allows to obtain the cross-sectional forces and moments and the reduced tangential tensors.

Fig. 5 shows the flow chart of the cross-sectional analysis procedure for a cross-section with $N_{\text {fiber }}$ fibers and $k$ simple components associated to each fiber. This method avoids the formulation of constitutive laws at cross-sectional level. Different types of inelasticity such as visco damage or plasticity are captured by means of the constitutive laws of the components of the composite of each fiber. Each cross-section is associated to the volume of a part of the beam and, therefore, constitutive nonlinearity at element level is captured through the cross-sectional integration.

Arbitrarily shaped cross-sections can be analyzed by means of the proposed integration method. However, the mechanical equilibrium at element level does not implies mechanical equilibrium among fibers in the inelastic range due to the fact that the present beam model solves the constitutive equations for each fiber independently of the behavior of the contiguous ones.
If materials presenting softening are associated to the fibers, the strain localization phenomenon can occur on specific integration points on the beam for certain loading levels $[36,37]$. Softening behavior of fibers imply the induction of a softer response at cross-sectional level and, in this manner, the strain localization induced at material point level is translated to the cross-sectional force-displacement relationships. In general, the structural response becomes dependent on the mesh size. In this work, the mesh independent response of the structure is obtained regularizating the energy dissipated on each fiber and limiting this value to the specific fracture energy of the material. Details about the regularization process can be consulted in $[35,39]$. However, other alternative procedures based on considering strong discontinuities on the generalized displacement field of the beam can be consulted in [3-5].

The estimation of the FPK stress vector at any material point on the cross-section gives a suitable starting point for defining a damage index representative for the real remaining loading capacity of a structure [16,28]. The damage index developed in this work is based on the damage index given in Ref. [35] for the static case. A measure of the damage level of a material point can be obtained as the ratio of the existing stress level to its visco elastic counter part. Following this idea, it is possible to define the fictitious damage variable $\mathrm{D}$ as follows:

$\sum_{i}\left|P_{1 i}^{\mathrm{mt}}\right|=(1-\check{D}) \sum_{i}\left|P_{1 i 0}^{\mathrm{mt}}\right|=\longrightarrow \check{D}=1-\frac{\sum_{i}\left|P_{1 i 0}^{\mathrm{mt}}\right|}{\sum_{i}\left|P_{1 i 0}^{\mathrm{mt}}\right|}$,

where $\left|P_{1 i}^{\mathrm{mt}}\right|$ and $\left|P_{1 i 0}^{\mathrm{mt}}\right|$ are the absolute values of the components of the existing (rate dependent) and visco elastic stress vectors, respectively. It is worth to note that $\check{D}$ is a measure of the remaining load carrying capacity. Initially, $\check{D}=0$ but when the entire fracture energy of the material is dissipated $\left|P_{1 i}^{\mathrm{mt}}\right| \rightarrow 0$ and, therefore, $\check{D} \rightarrow 1$.

Eq. (43) can be applied to consider a structural member, a part of the structure or the whole structure, by integrating the stresses over the corresponding volume, $\bar{V}$ of the structure. In this manner, local and global damage indices are calculated: 


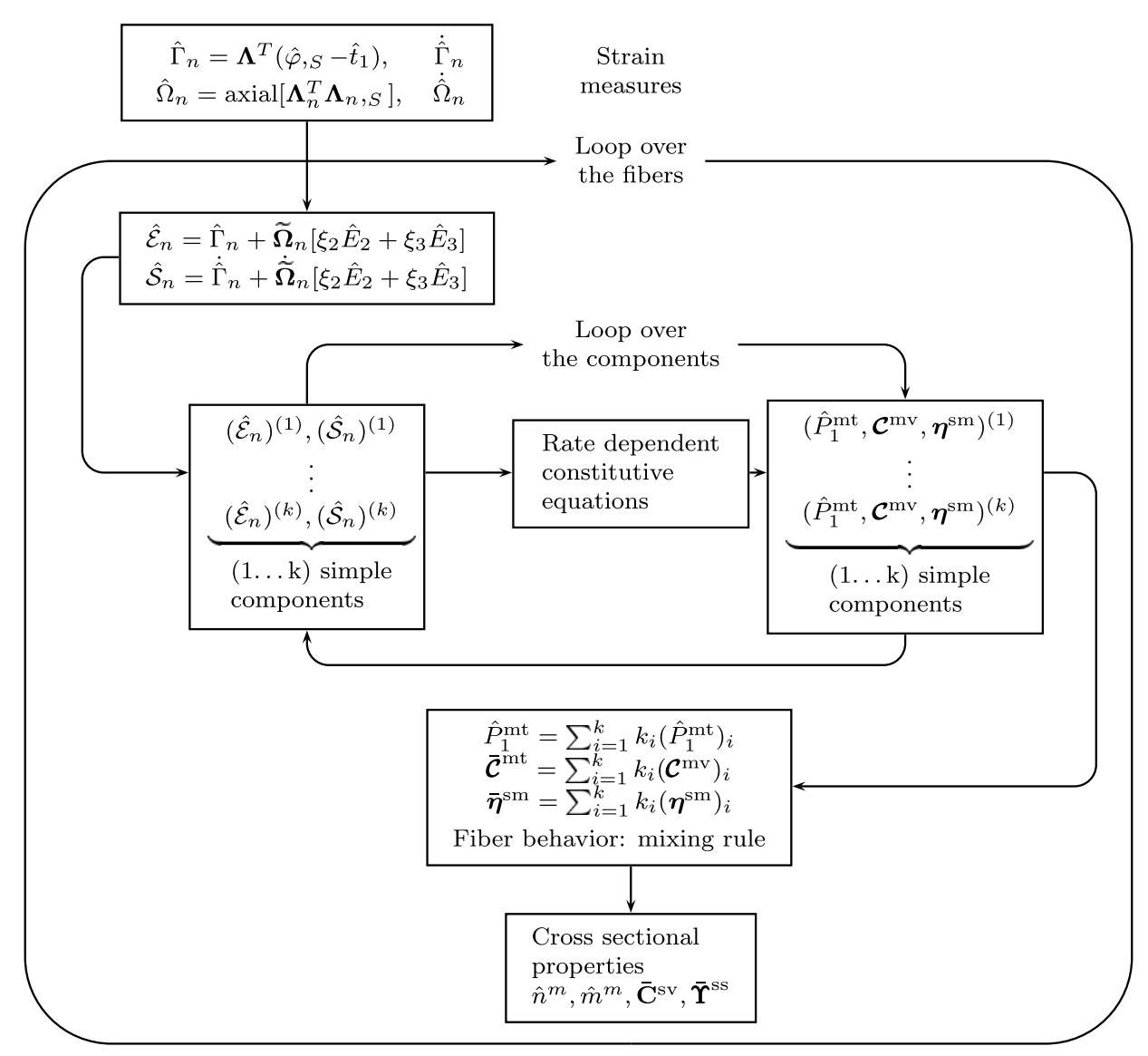

Fig. 5. Flow chart of the cross-sectional integration.

$\check{D}=1-\frac{\int_{\bar{V}}\left(\sum_{i}\left|P_{1 i 0}^{\mathrm{m}}\right|\right) \mathrm{d} \bar{V}}{\int_{\bar{V}}\left(\sum_{i}\left|P_{1 i 0}^{\mathrm{m}}\right|\right) \mathrm{d} \bar{V}}$

\section{Numerical examples}

\subsection{Flexible beam in helicoidal motion}

The example corresponds to the validation of the proposed formulation for the elastic case. For comparative purposes, an example of Ref. [26] has been reproduced here. It corresponds to the helicoidal motion of a straight beam constrained to slide and rotate along the vertical axis $Z$ of Fig. 6. A constant vertical load and torque is applied during $2.5 \mathrm{~s}$ as indicated in the same figure. Due to the fact that it is a purely elastic example, no cross-sectional integration is required and the mechanical properties are taken from [26] as: $E \mathscr{A}=G \mathscr{A}=10^{4}, E \mathbf{I}=G \boldsymbol{J}=10^{3}, \mathscr{A}_{\rho}=1.0$ and $\boldsymbol{J}_{\rho}=\operatorname{Diag}[20,10,10]$, where $E$ and $G$ are the Young and shear elastic modulus and $\mathscr{A}, \mathbf{I}, \boldsymbol{J}, \mathscr{A}_{\rho}$ and $\boldsymbol{J}_{\rho}$ are the cross-sectional area, the second moment of inertia, the torsional inertia, the beam mass and the inertial dyadic per unit of length, respectively. Ten linear beam elements and a time-step size $\Delta t=0.5 \mathrm{~s}$ were used in the numerical simulations.

The results of the numerical simulations are presented in Fig. 7 which are in good agreement with those given in [26] for the three components of the displacement of the free end of the beam.

\subsection{Visco elastic right angle cantilever beam}

The right angle cantilever beam shown in Fig. 8a is dynamically loaded by an out of plane concentrated force of $250 \mathrm{~N}$ at the elbow. The shape and duration of the applied load is show in the same figure. The total duration of the analysis is $4.5 \mathrm{~s}$, which includes the period of time when the load is being applied and the following free
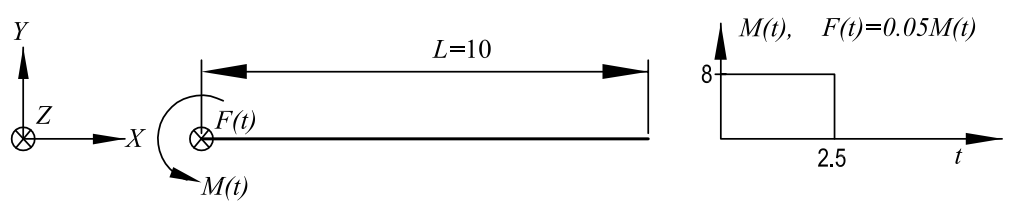

Fig. 6. Flexible beam in helicoidal motion. 

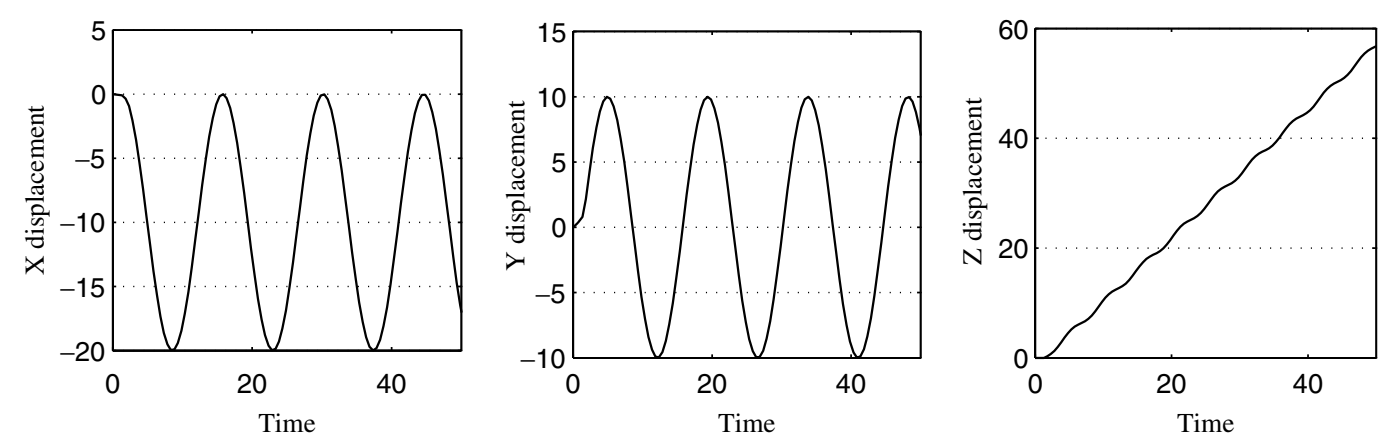

Fig. 7. Displacements time history response of the free end of the beam.

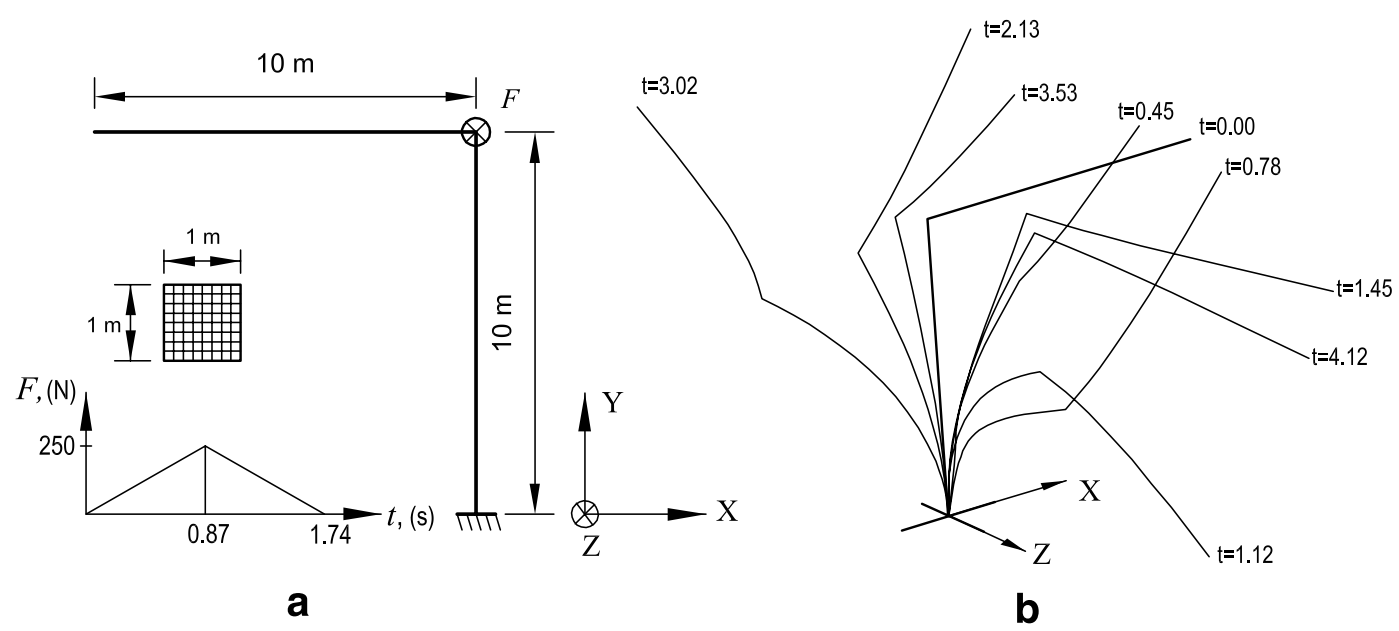

Fig. 8. Right angle frame. (a) A concentrated out of plane load is applied on the elbow node. (b) Deformed configurations for different time steps for the case of the undamped system.

vibration of the system. The time step is $0.03 \mathrm{~s}$. The mechanical properties are: an elastic modulus of $5.0 \times 10^{4}$ $\mathrm{N} \mathrm{mm}^{-2}$, a Poisson coefficient of 0.2 and a mass density of $0.1 \mathrm{~kg} \mathrm{~mm}{ }^{-3}$. Three quadratic elements with two Gauss integration points have been used for each structural member. The cross-section is meshed into a grid of $8 \times 8$ equally spaced quadrilaterals equipped with one integration point. Several deformed shapes of the system are show in Fig. 8b for the undamped elastic case. It is interesting to note that the motion of the system involves large torsion and bending and the magnitude of the displacements is of the same order as the dimensions of the undeformed geometry.

Three values for the viscosity $\eta=0.01,0.03$ and $0.04 \mathrm{~s}$ are used in the numerical simulations, along with the visco elastic constitutive law, in order to highlight the effects of the damping on the behavior of the system. Figs. 9a and $9 \mathrm{~b}$ show the time history of the displacement of the tip and the elbow in $\mathrm{Y}$ direction for the undamped system and for the three values of the viscosity. It is possible to see from these figures that increasing values of the viscosity contributes to decrease the maximum displacements of the system during the nonlinear oscillations.

Viscosity also contributes to dissipate the high frequency content in the response, what can be seen due to the fact that increasing values of the parameter $\eta$ imply smoother time history responses. Finally, a more significative appreciation of the effects of viscosity can be obtained from Fig. 10, where the time history of the displacements of the tip in the $Y-Z$ plane is shown. It is possible to see in this figure, that the increment of the value of $\eta$ diminishes the amplitude of the motion of the mechanical system.

\subsection{Near resonance response of a composite cantilever beam}

This example studies the cantilever beam subjected to a sinusoidal base acceleration shown in Fig. 11. Different constitutive behaviors for the material are considered: (i) visco elastic with different viscosity values; (ii) a visco damage model; (iii) a composite material with two simple constituents: a visco damage phase and a rate independent plastic phase, both of them with a volumetric fraction of $50 \%$. The elastic properties of the three materials are the same, excepting the fracture energy (see Table 2). The beam has been modeled using 20 quadratic elements and a reduced integration scheme is used in order to avoid shear locking [50]; the beam cross-section has been meshed into a grid of $8 \times 8$ quadrilaterals with four integration points in each of them. A linear modal analysis of the beam model gives a fundamental period of $1.77 \mathrm{~s}$ and the second and third modes have periods of 0.27 and $0.099 \mathrm{~s}$, respectively. 

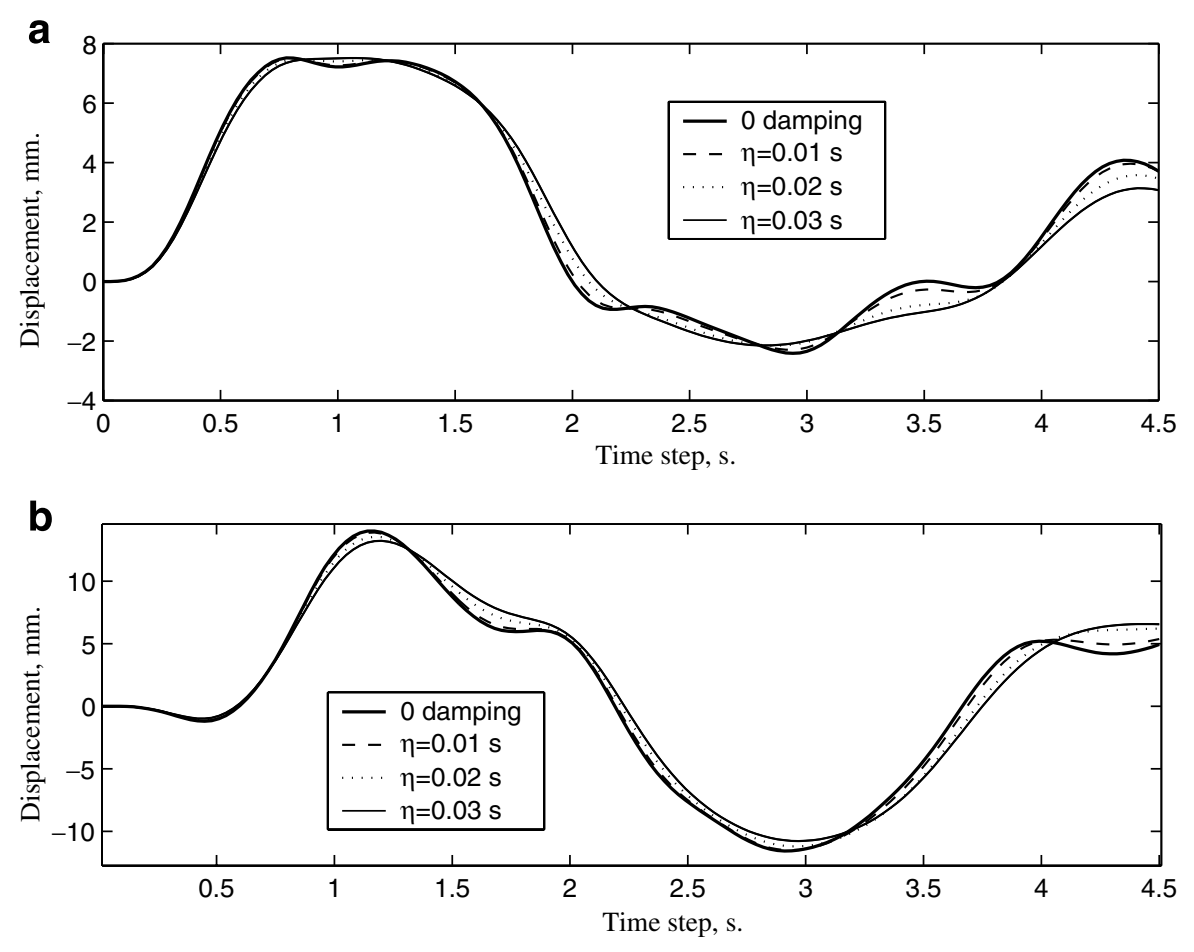

Fig. 9. Displacement time history responses in the $Y$ direction. (a) Tip. (b) Elbow.

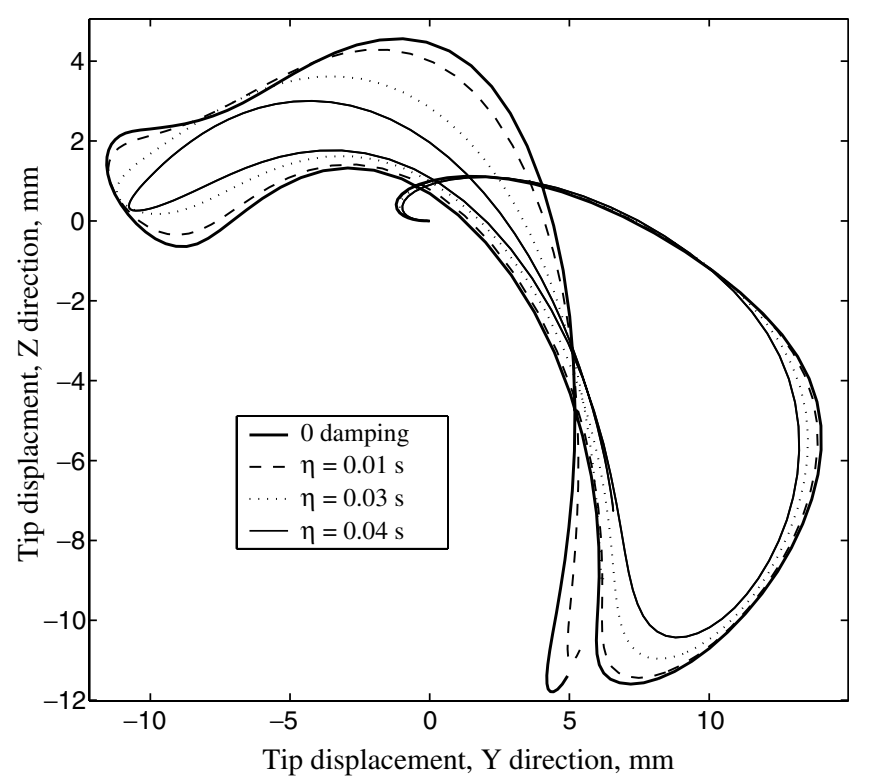

Fig. 10. Tip displacements time history response in the $Y-Z$ plane.

The dynamic nonlinear response of the beam is obtained for a sinusoidal excitation with a frequency of $1.75 \mathrm{~s}$ aimed to induce near-resonance effects in the first elastic mode. The time step is $0.03 \mathrm{~s}$. Three values for viscosity have been considered: $0.0,0.01,0.025$ s. Fig. 12 shows the displacement time history of the top node in the horizontal and vertical directions. Important reductions in the amplitude of the oscillations are obtained as the viscosity is increased. For the value of $\eta=0.025 \mathrm{~s}$ almost all the second order effects are suppressed due to reductions of the dynamic

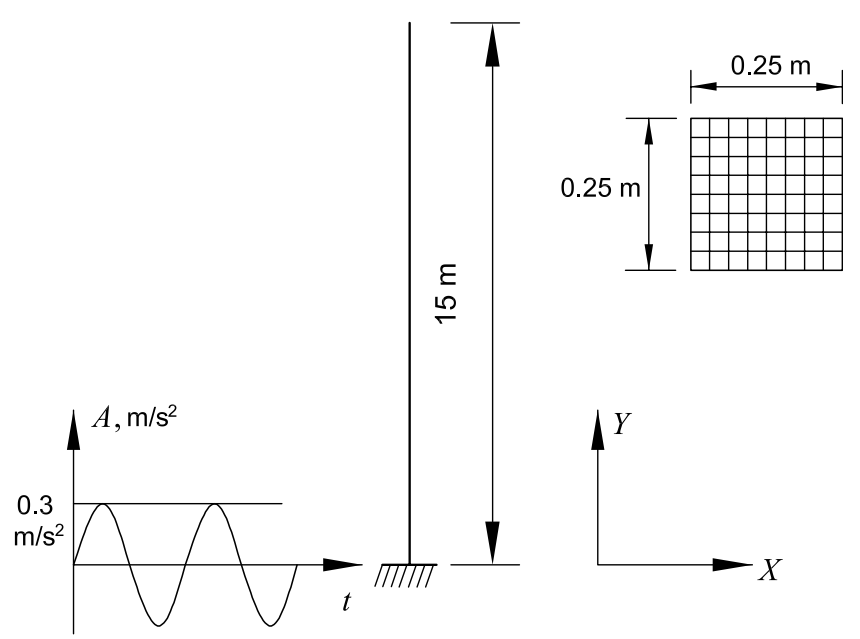

Fig. 11. Vertical cantilever beam subjected to a sinusoidal base acceleration.

Table 2

Mechanical properties

\begin{tabular}{lcccccc}
\hline & $E(\mathrm{MPa})$ & $v$ & $\begin{array}{l}f_{\mathrm{c}} \\
(\mathrm{MPa})\end{array}$ & $n$ & $\begin{array}{l}G_{\mathrm{f}} \\
\left(\mathrm{N} \mathrm{mm}^{-2}\right)\end{array}$ & $\begin{array}{l}\rho_{0} \\
\left(\mathrm{~kg} \mathrm{~mm}^{-3}\right)\end{array}$ \\
\hline Rate dependent & $2.5 \times 10^{4}$ & 0.20 & 25 & 1 & 25 & $2.4 \times 10^{-9}$ \\
Rate independent & $2.5 \times 10^{4}$ & 0.15 & 500 & 1 & 5000 & $2.4 \times 10^{-9}$ \\
\hline
\end{tabular}

amplification of the response. Vertical displacements are reduced from $400 \mathrm{~mm}$ for the undamped case to $12 \mathrm{~mm}$.

As it has been mentioned, due to the change of configuration of the structure near the resonance, the displacements in the vertical direction are amplified and, 

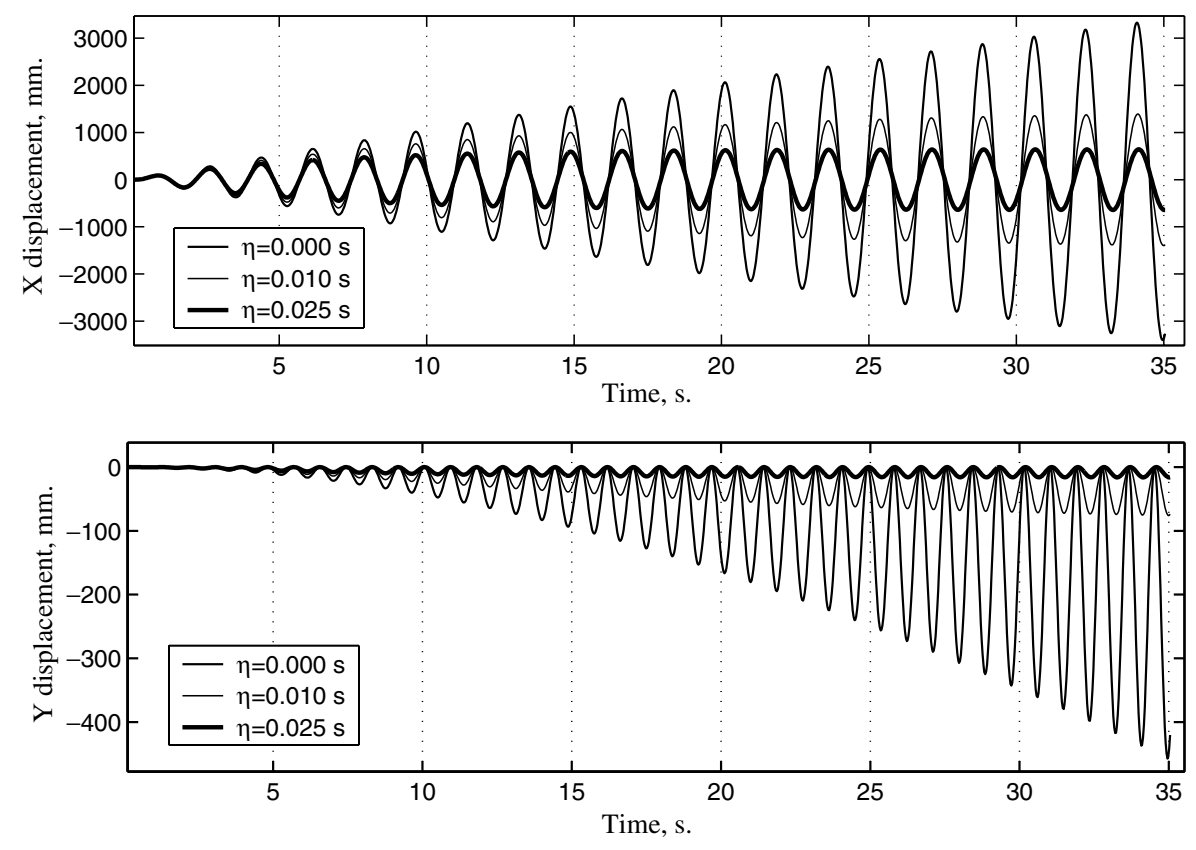

Fig. 12. Horizontal and vertical displacement at the top.

therefore, axial forces with high frequency content appear in the response.

As stated in Ref. [20], the suppression of high frequency content becomes a desirable feature of a nonlinear time stepping scheme. In the present formulation, the reduction of the contribution of the higher modes to the response is obtained by adding viscous mechanisms to the constitutive laws. In Fig. 13, the beam structure is subjected to a sinusoidal base acceleration record with a period of $0.1 \mathrm{~s}$ acting during $2.5 \mathrm{~s}$ followed by a free vibration of $2.5 \mathrm{~s}$. The time step is $0.005 \mathrm{~s}$. The horizontal displacement response of the top node of the system is depicted in Fig. 13a where it is possible to see that the a coupled response corresponding to the first and third modes dominates the structural behavior. Amplification of the third mode is observed. It is also possible to appreciate in this figure that increments in the values of the viscosity $(\eta=0.000,0.001,0.005 \mathrm{~s})$ have the effect of reducing mainly the vibration associated to the higher modes. This result is in good agreement with the solution proposed in [20] in the sense that dissipative mechanisms, for time stepping schemes, based on the strain rate or velocity contributes to eliminate the high frequency content in the response. Fig. 13b shows the temporal evolution of the energy dissipation which is a scalar quantity
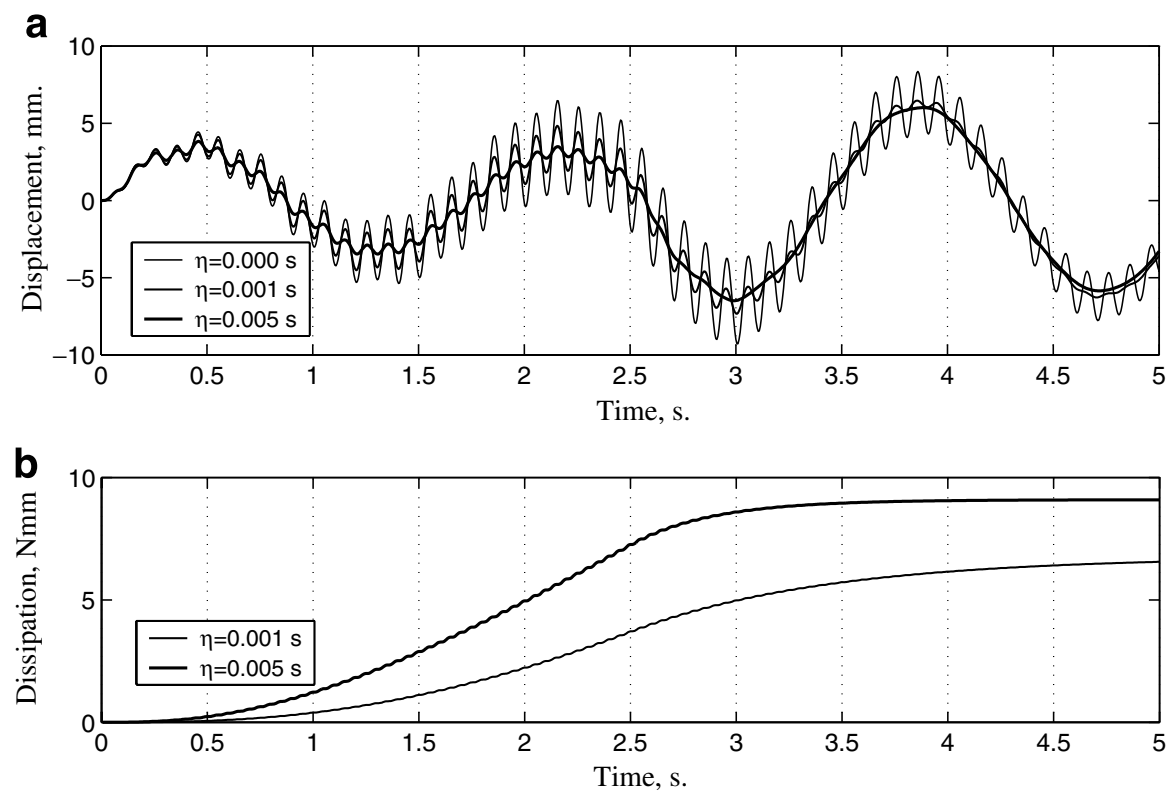

Fig. 13. (a) High frequency content suppression. (b) Energy dissipation. 
calculated from Eq. (20) at constitutive level and then integrating over the volume of the structure. It is possible to see that higher values of $\eta$ implies higher dissipation rates and the stabilization of the response is achieved before.

Finally, the cantilever beam is subjected to a sinusoidal base acceleration with a period of $1.75 \mathrm{~s}$ and a duration of $35 \mathrm{~s}$ in order to induce near-resonance response. The time step is $0.03 \mathrm{~s}$. The dynamic response was obtained for three constitutive options: the first one corresponds to the visco damage model with $\eta=0.001 \mathrm{~s}$, the second one is the elastic plastic model, where the energy dissipation is obtained by displacement dependent (no viscous) mechanisms and the third one is a composite material with a $50 \%$ of the first material and a $50 \%$ of the second one.

Fig. 14a compares the response for the viscous damage model with the undamped elastic case. It is possible to see that the change of the mechanical properties of the material due to the progressive damage induces stiffness degradation, changing the instantaneous dynamical properties of the structure. In this case, the period of the structure is increased due to the fact that the damage auto decouples the structural response from resonance. Dissipation due to damage is added to the viscous one and, therefore, extra energy dissipation contributes to reduce the dynamic response.

Fig. 14b shows comparative results for the time history of the three constitutive behaviors. It is worth noting that, in the case of the composite material, when most of the material corresponding to the visco damage model has been degraded in the softening zone [35], the response of the structure is controlled by the plastic behavior. After a degrading phase between the 3 and $13 \mathrm{~s}$ the response of the composite coincides with that corresponding to the purely plastic model. Finally, Figs. $15 \mathrm{a}$ and $15 \mathrm{~b}$ show the evolution of the global damage indices and dissipated energy for the three different constitutive options.

\subsection{Nonlinear vibration of a spatial curved structure}

The 3D structure shown in Fig. 16 is an open ring located in the $X-Y$ plane, which has a fixed end and the another one is connected to a straight column lying on the $X-Z$ plane. Two loads are applied on the points $A$ and $B$ in the $X$ and $Z$ directions, respectively. The shape and duration of the loading is given in the same figure. The loading step is $0.01 \mathrm{~s}$. The mechanical properties of the materials are: $E=5 \times 10^{4} \mathrm{~N} \mathrm{~mm}^{-2}, v=0.0, G_{\mathrm{f}}=$ $1000 \mathrm{~N} \mathrm{~mm}^{-2}, n=1, \rho_{0}=1 \times 10^{-8} \mathrm{~kg} \mathrm{~mm}^{-3}$ and $f_{\mathrm{c}}=8$ $\mathrm{MPa}$. Three cases are studied: (i) Elastic case. (ii) Visco damage model with $\eta=0.004 \mathrm{~s}$. (iii) Rate independent plasticity with $G_{\mathrm{f}}=1 \times 10^{7} \mathrm{~N} \mathrm{~mm}^{-2}$. The same threshold limit is used for the models (ii) and (iii).

Twenty quadratic, initially curved elements are used for the ring and ten quadratic elements for the column considering two Gauss integration points. The beam cross-section is meshed into a $8 \times 8$ grid of equally spaced quadrilaterals with one integration point per fiber.

Due to the directions of the applied loads and the development of inertial forces, each member of the structure is subjected to a complex state of internal stresses including torsion, flexion extension and shearing. Figs. 17 and 18 show the nonlinear time history responses for the three components of the displacement of the points A and B of Fig. 16. It is worth to note that the displacements of the
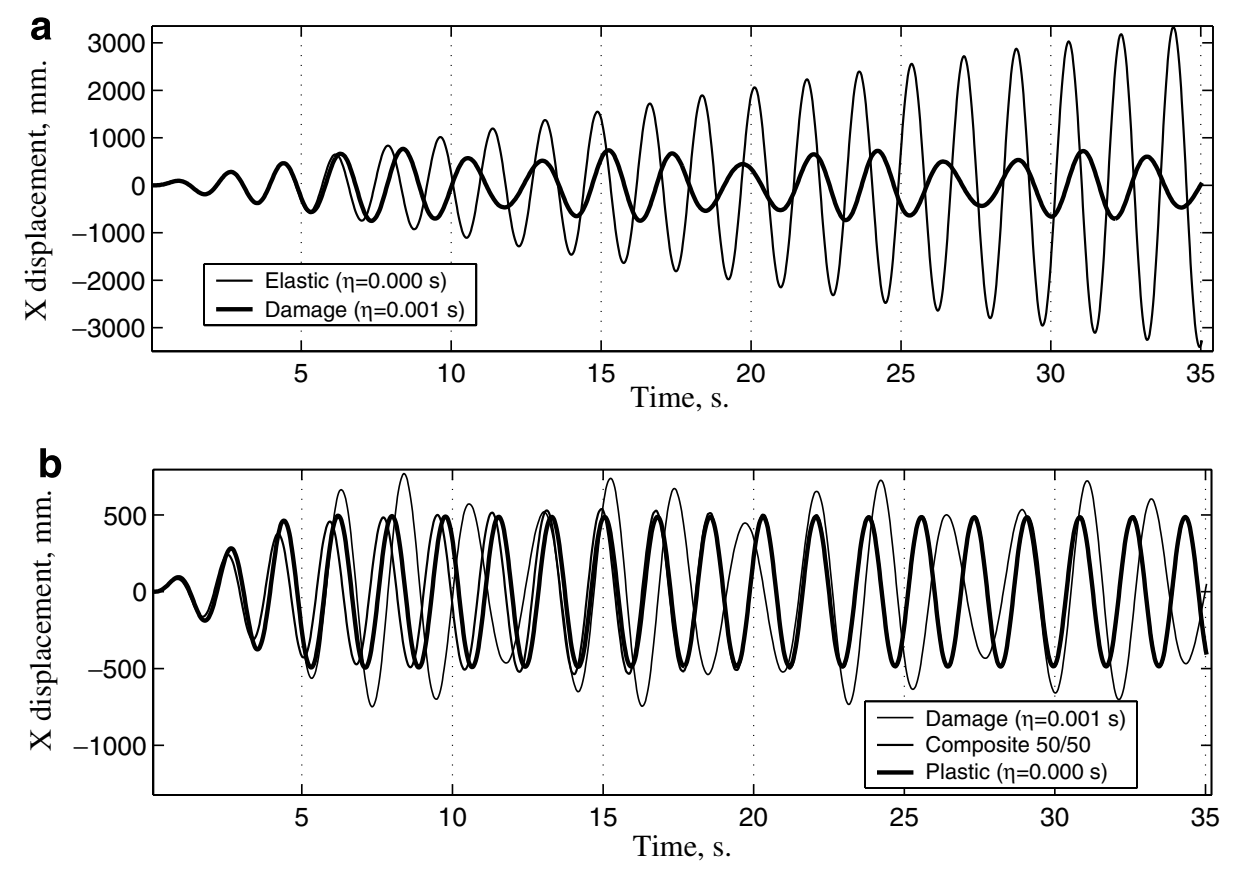

Fig. 14. Coupled geometric and constitutive nonlinear dynamic responses. (a) Undamped elastic response compared with a visco damage constitutive model. (b) Visco damage, plastic and composite responses. 
a

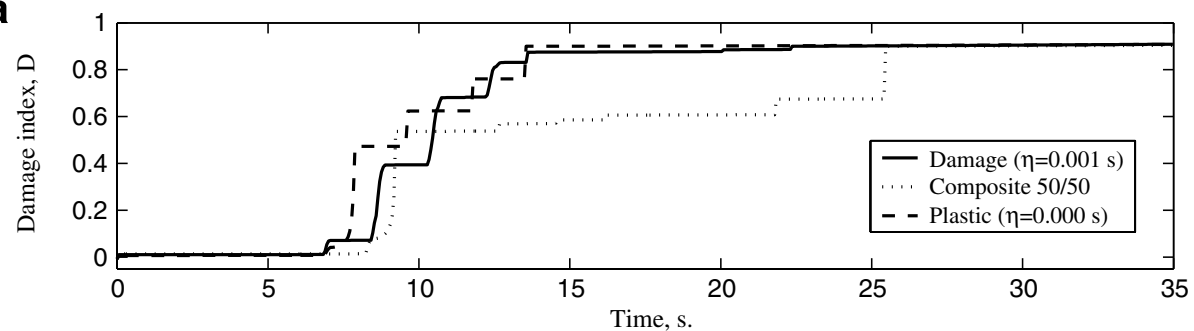

b

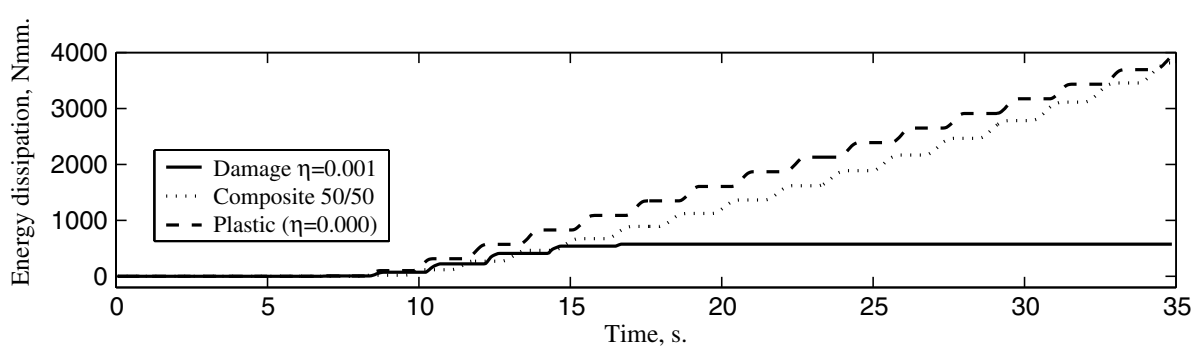

Fig. 15. (a) Evolution of the global damage index during the dynamic response. (b) Evolution of the energy dissipation.

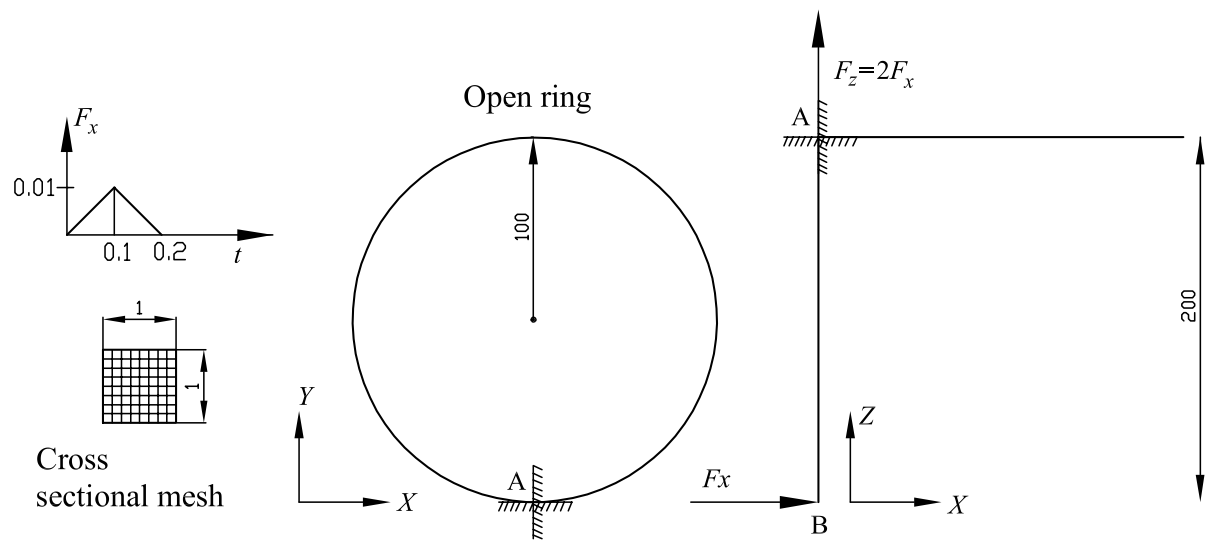

Fig. 16. Three-dimensional curved beam structure.

systems with inelastic behavior are greater than the elastic case due to the fact that the initial loading induces degradation and plasticity and the structure needs to develop more displacement for finding a configuration stable with the new loading state. In the plastic case the structure finally vibrates about a configuration which includes permanent deformation as it can be evidenced from the $Z$ component of both figures.

\subsection{Study of a RC beam structure}

This example studies of the fully geometric and constitutive static and dynamic behavior of the RC beam structure shown in Fig. 19. The structure was seismically designed according to the Eurocode 8, for firm soil, a base acceleration of $0.4 \mathrm{~g}$ and a ductility reduction factor of 6 . The distribution of steel reinforcements are given in the same figure and Table 3. The precise analysis of the response allows to carry out a safer determination of the seismic design parameters, such as: damage, ductility, overstrength, collapse load, inter story drifts, energy dissipation capacity, etc. $[13,28]$. The study of the time evolution of the local damage index throughout the structural elements provides relevant information about the structural zones where ductility demand and energy dissipation are concentrated. These results allow validating and improving the engineering design of buildings located in high seismic areas.

The model is developed using four quadratic elements with two Gauss integration points in each structural element. Most of the mechanical properties of the materials are given in Table 4 . Each cross-section is meshed into a grid of $8 \times 8$ quadrilaterals with one integration point by fiber. The tensile fracture energy of the concrete takes values between 3.0 and $6.0 \mathrm{~N} \mathrm{~mm}^{-2}$, corresponding to different steel ratios of the stirrups. The mass density of the concrete of the beams is modified in order to consider the mass contribution of the dead and live loads acting at the corresponding floor level. A viscosity value of $\eta=0.001 \mathrm{~s}$ has been supposed for the concrete. A linear modal analysis reveals that the periods of the first four modal shapes are: $1.20,0.36,0.19$ and $0.12 \mathrm{~s}$, respectively. 

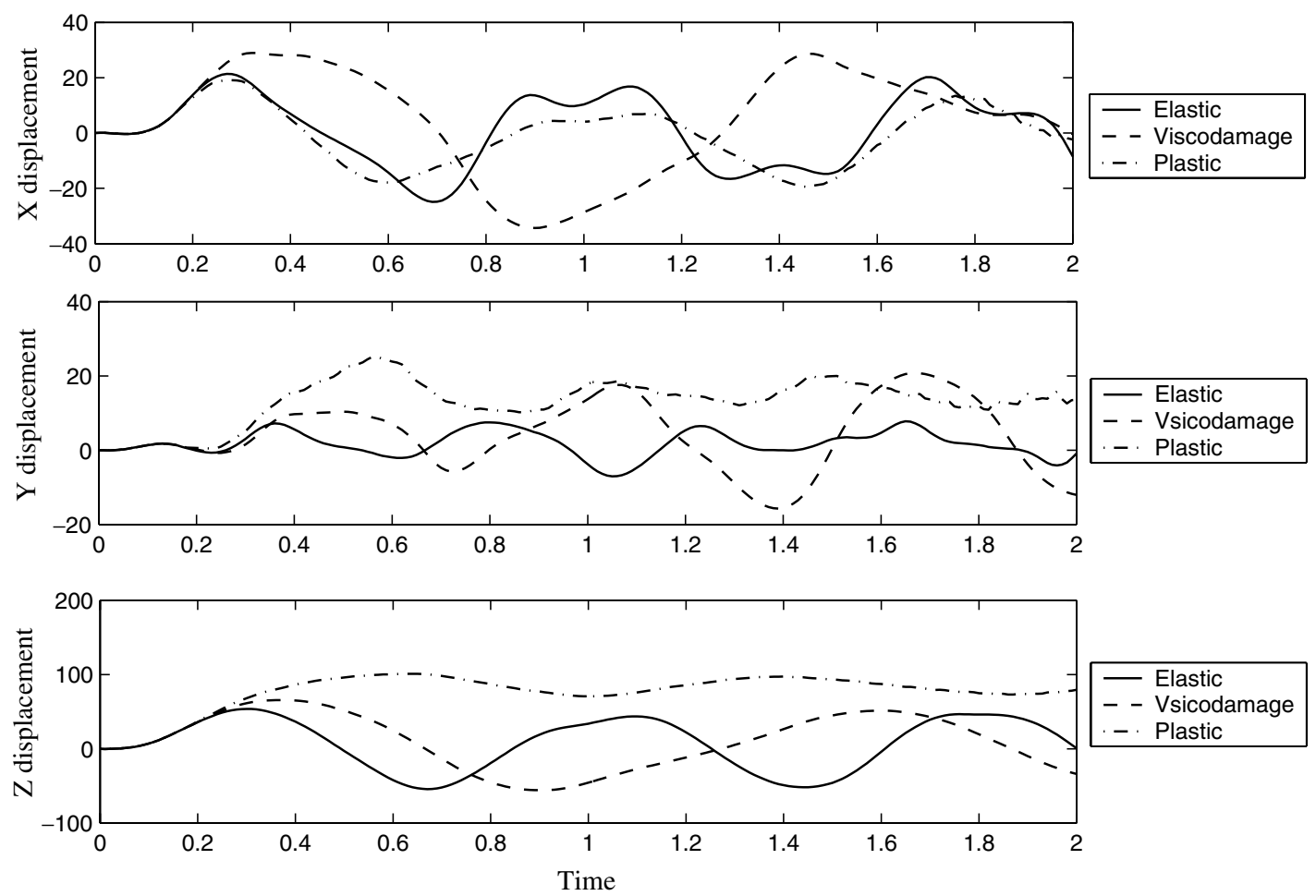

Fig. 17. Displacement time history response of the node A.
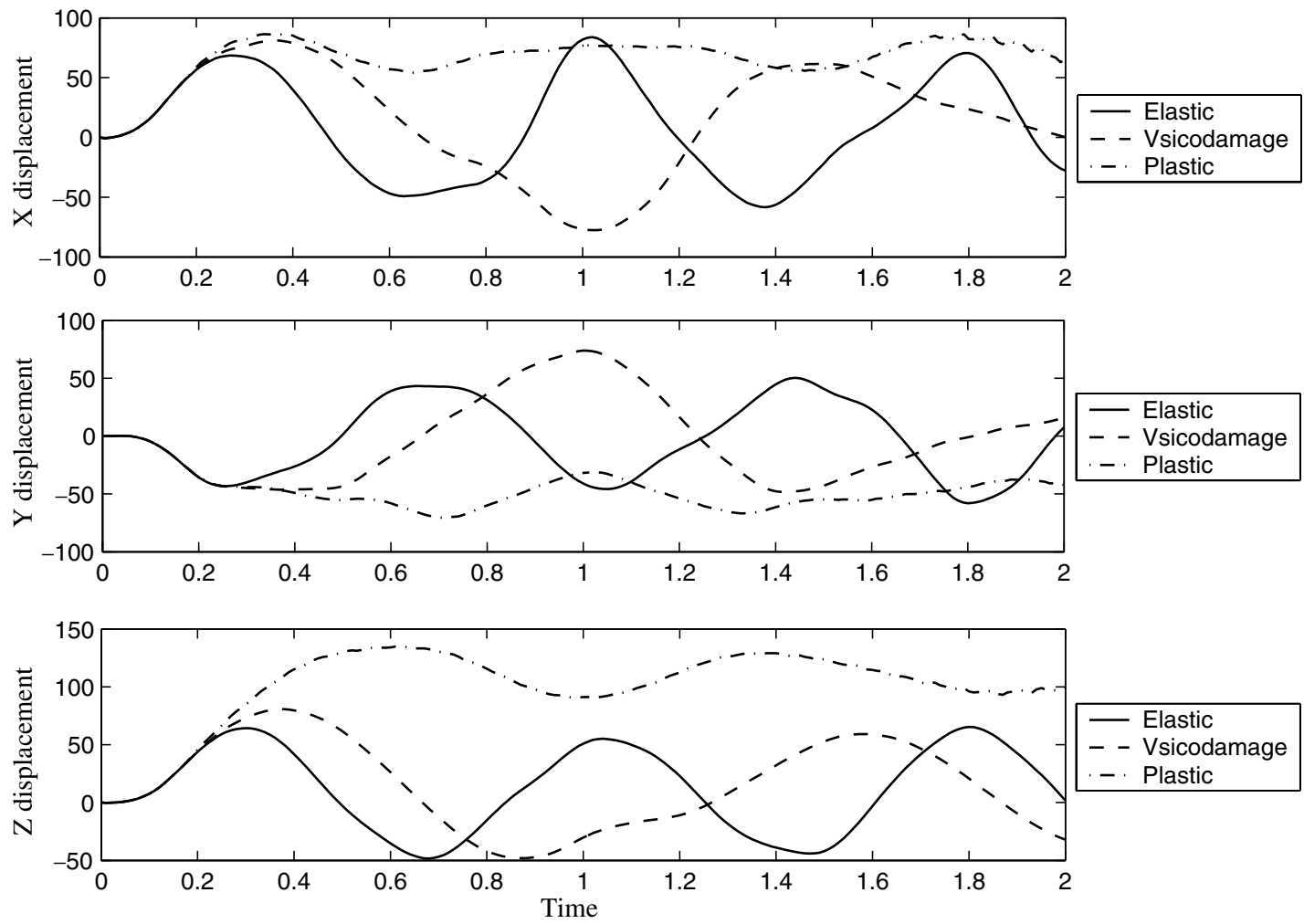

Fig. 18. Displacement time history response of the node B.

Firstly, a static pushover test using the inverted triangular loading path is performed in order to obtain the charac- teristic capacity curve of the structure expressed in terms of the horizontal displacement of the left top corner node and 

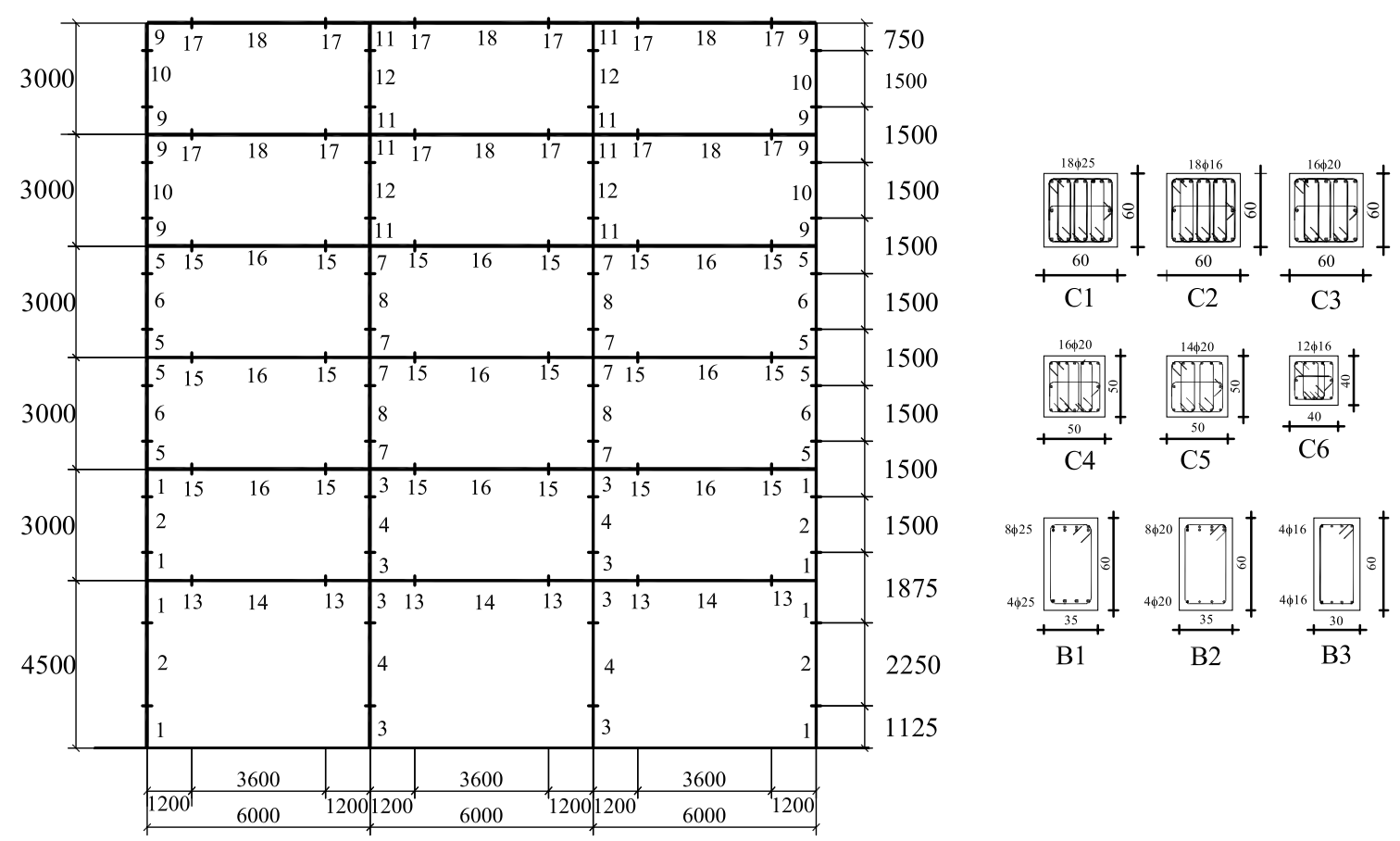

Fig. 19. Structural model of the RC planar frame.

Table 3

Steel reinforcement details of the different zones of the building

\begin{tabular}{|c|c|c|c|c|c|c|}
\hline Zone & 1 & 2 & 3 & 4 & 5 & 6 \\
\hline $\begin{array}{c}\text { Section } \\
\text { type }\end{array}$ & $\mathrm{C} 1$ & $\mathrm{C} 1$ & $\mathrm{C} 2$ & C2 & C3 & C3 \\
\hline Stirrup & $\begin{array}{l}\phi \\
12 @ 12\end{array}$ & $\begin{array}{l}\phi \\
12 @ 15\end{array}$ & $\begin{array}{l}\phi \\
12 @ 12\end{array}$ & $\begin{array}{l}\phi \\
12 @ 15\end{array}$ & $\begin{array}{l}\phi \\
12 @ 12\end{array}$ & $\begin{array}{l}\phi \\
12 @ 15\end{array}$ \\
\hline Zone & 7 & 8 & 9 & 10 & 11 & 12 \\
\hline $\begin{array}{c}\text { Section } \\
\text { type }\end{array}$ & $\mathrm{C} 4$ & $\mathrm{C} 4$ & $\mathrm{C} 5$ & $\mathrm{C} 5$ & C6 & C6 \\
\hline Stirrup & $\begin{array}{l}\phi \\
12 @ 12\end{array}$ & $\begin{array}{l}\phi \\
12 @ 15\end{array}$ & $\begin{array}{l}\phi \\
10 @ 12\end{array}$ & $\begin{array}{l}\phi \\
10 @ 15\end{array}$ & $\begin{array}{l}\phi \\
10 @ 12\end{array}$ & $\begin{array}{l}\phi \\
10 @ 15\end{array}$ \\
\hline Zone & 13 & 14 & 15 & 16 & 17 & 18 \\
\hline $\begin{array}{c}\text { Section } \\
\text { type }\end{array}$ & B1 & B1 & B2 & B2 & B3 & B3 \\
\hline Stirrup & $\begin{array}{l}\phi \\
12 @ 12\end{array}$ & $\begin{array}{l}\phi \\
12 @ 20\end{array}$ & $\begin{array}{l}\phi \\
12 @ 12\end{array}$ & $\begin{array}{l}\phi \\
12 @ 20\end{array}$ & $\begin{array}{l}\phi \\
10 @ 12\end{array}$ & $\begin{array}{l}\phi \\
10 @ 20\end{array}$ \\
\hline
\end{tabular}

Table 4

Mechanical properties of the materials

\begin{tabular}{lrlclc}
\hline & $E(\mathrm{MPa})$ & $v$ & $f_{\mathrm{c}}(\mathrm{MPa})$ & $n$ & $G_{\mathrm{f}}\left(\mathrm{N} \mathrm{mm}^{-2}\right)$ \\
\hline Concrete & 21,000 & 0.20 & 25 & 8 & 1 \\
Steel & 200,000 & 0.15 & 500 & 1 & 500 \\
\hline
\end{tabular}

the horizontal reaction on the supports. Even if capacity curves are widely accepted as valid substitutes of time history analysis, the question about if dynamic or increasing load paths are more convenient to obtain capacity curves, stays still opened [13]. Secondly, the structure is subjected to an increasing sinusoidal base acceleration of period

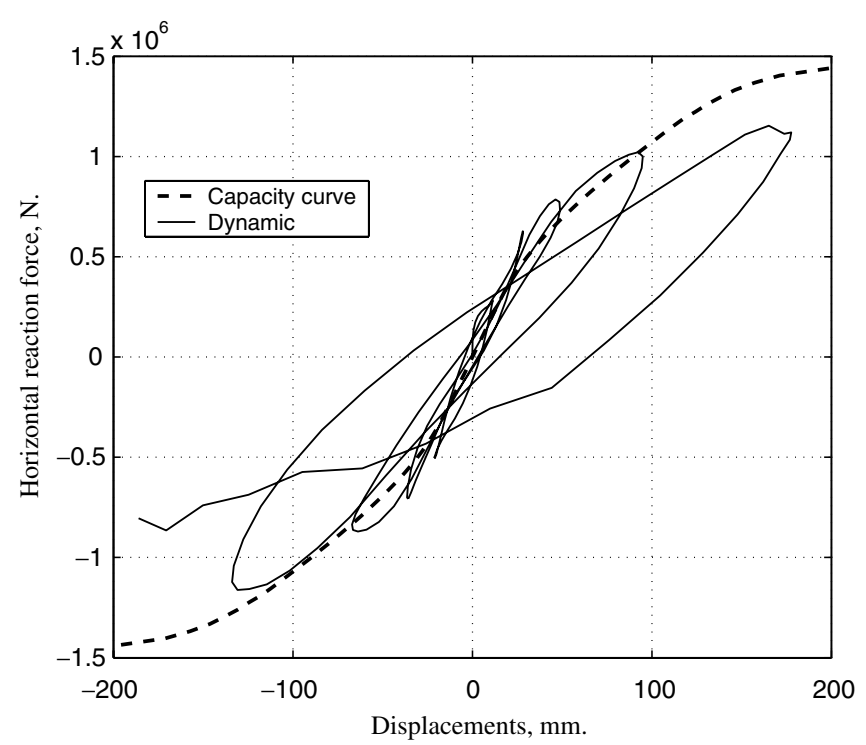

Fig. 20. Horizontal displacements versus base reaction for the static pushover and the dynamic analysis.

$1.2 \mathrm{~s}$ for inducing a near to resonance response, with minimum and maximum values of acceleration of 50 and $200 \mathrm{~mm} \mathrm{~s}^{-2}$, respectively. The time step is $0.04 \mathrm{~s}$.

The capacity curve obtained by means of the pushover analysis and the hysteretic cycles in the dynamic case are superposed in Fig. 20, where it is possible to see that the capacity curve underestimate the real response of the structure for the low amplitude hysteretic cycles. After an important degradation of the concrete has occurred, the hysteretic cycles are enclosed by the capacity curve. This 
fact justifies the employment of pushover response curves for predicting the seismic response of regular buildings, due to the fact that frequently, during a seismic action, the first movements contribute to the initial cracking of the concrete and, when the strongest vibrations have place, the response of the structure should be limited by the capacity curve case. The global ductility value, esti- mated from the capacity curve is about 6 , in good agreement with the hypothesis of design.

The evolution of the local damage indices provides more precise information about the ductility demand and energy dissipation demand for the principal structural members. Fig. 21a shows the evolution of the local damage index for the static case. It is possible to see that the nonlinear a
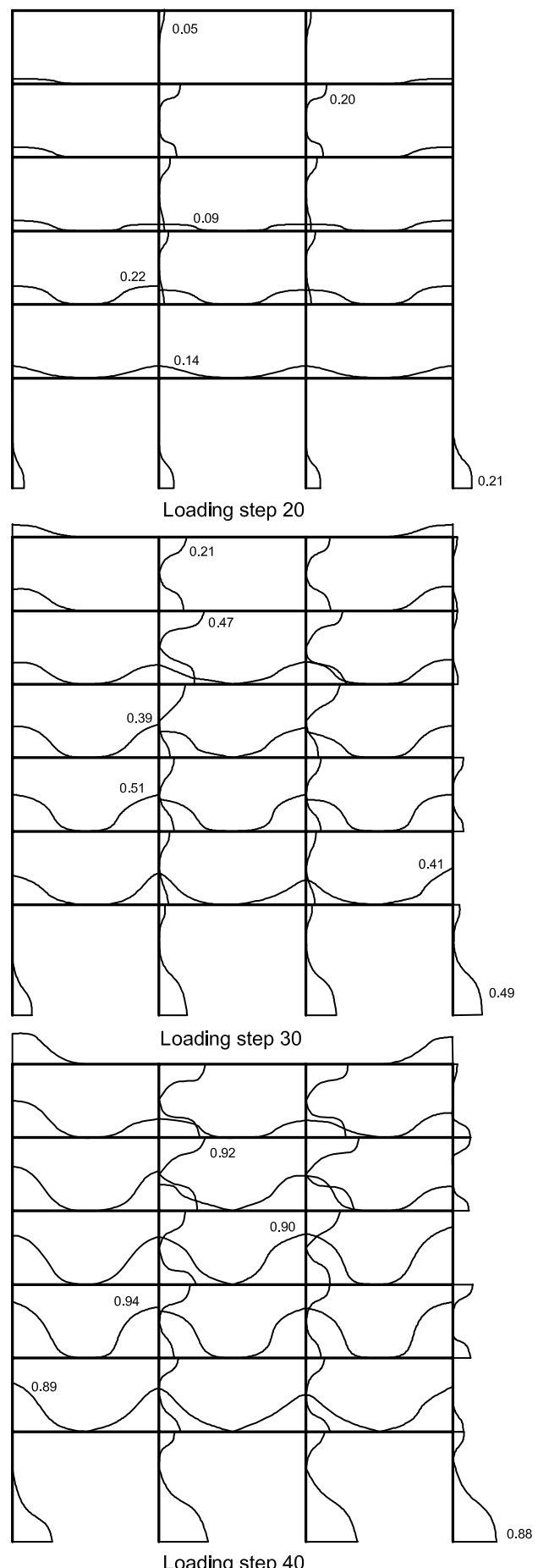

b

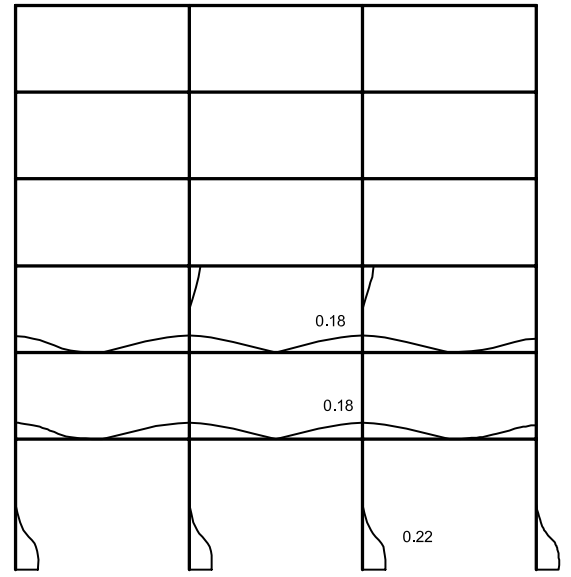

Time step $3.6 \mathrm{~s}$
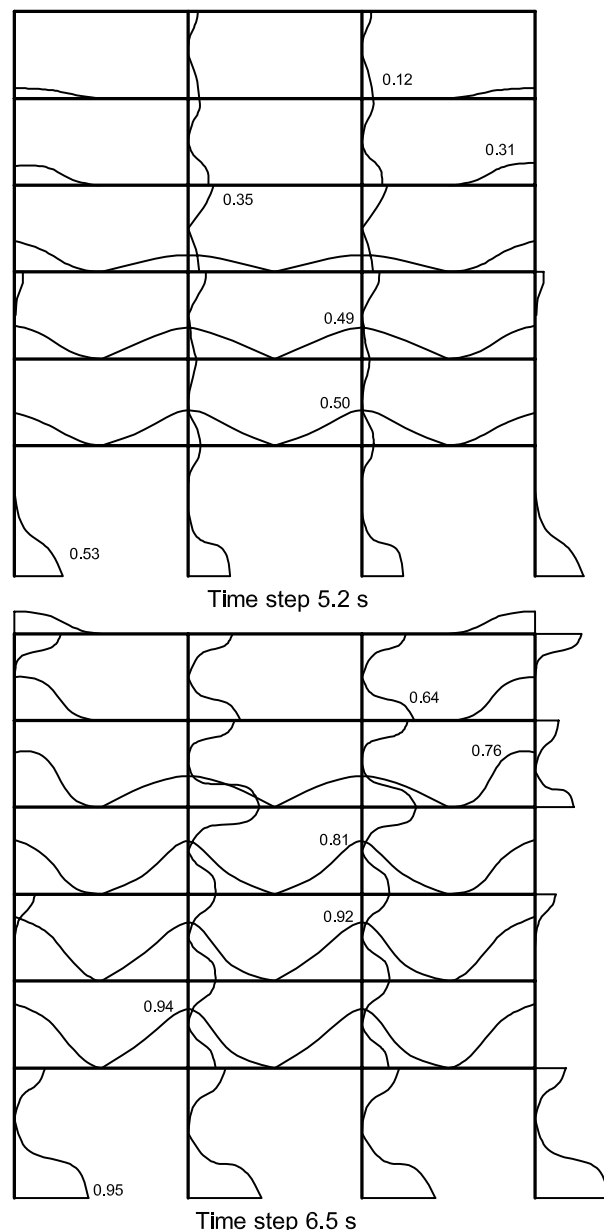

Fig. 21. Evolution of the local damage indices. (a) Pushover analysis. (b) Dynamic case. 
behavior is concentrated mainly in the beam elements of the first three floors and in the base columns. This result is in good agreement with the design guidelines included in most of the modern seismic codes, that is, the building was designed with weak-beams and strong-columns aiming to dissipate the energy without affecting the global stability of the structure. Moreover, the concentration of damage at the base of the columns indicate that these members should be provided with extra reinforcements in order to obtain large inelastic incursions without brittle failures. The diagram of Fig. 21a shows the evolution of the damage index corresponding to the static case while Fig. $21 \mathrm{~b}$ shows the corresponding to the dynamic case. It is possible to see that the failure mechanisms are similar with energy dissipation concentrated in the beams and in the base of the columns.

It is worth to note that even although the fiber model allows to predict complex nonlinear structural responses in both, static and dynamic cases, the numerical cost of the cross-sectional analysis can be significant when compared with the lumped nonlinear models. In this case, a typical cross-section meshed into a grid of $8 \times 8$ quadrilaterals with three materials requires solving $3 \times 8 \times 8$ constitutive equations. After that, a cross-sectional integration procedure is required in order to obtain the reduced forces and tangential tensors. In contrast, only one integration procedure is carried out for lumped models.

\section{Conclusions}

A geometrically exact formulation for initially curved and twisted beams in large displacements and rotations and finite strains has been extended in this work to consider arbitrary distributions of composite materials on the crosssections in the dynamic case. An explicit expression for the objective measure of the strain rate acting on each material point is deduced along with its linearized form. The linearization of the weak form of the momentum balance equations is carried out considering rate dependent inelasticity, which leads to the consistent deduction of the mass and viscous tangent stiffness tensors. The resulting model is implemented in a displacement based FE code.

A Newton-Raphson type of iterative scheme is used for the step-by-step solution of the discrete version of the linearized system. Due to the fact that the deformation map and their related dynamical variables belong to $\mathbb{R}^{3} \times S O(3)$, an appropriated version of Newmark's scheme is used and details about the numerical implementation of the iterative updating procedure are also addressed. It is worth to note that even though using small time steps the numerical dissipation can be reduced, additional benefits in the numerical performance could be obtained employing energy-momentum conserving schemes, at least in the conservative limit of the problems.

Each material point of the cross-section is assumed to be composed of several simple materials with their own constitutive laws. The mixing rule is used to describe the resulting composite. Viscosity is included at the constitutive level by means of a thermodynamically consistent visco damage model developed in terms of the material description of the FPK stress vector, which allows obtaining more realistic estimations of the dissipated energy. From the numerical point of view, the cross-sections are meshed into a grid of quadrilaterals, each of them corresponding to fibers directed along the axis of the beam. An additional integration loop, running on the number of fibers, is required to obtain the iterative cross-sectional forces, moments and the tangential stiffness tensors.

Local and global damage indices have been developed based on the ratio of the visco elastic to the nonlinear stresses. The proposed damage indices allow estimating the evolution of the global load carrying capacity of the structure and the damage state of the different members during dynamic actions.

The present formulation is validated by means of several numerical examples showing the ability of the present formulation for reproducing the mechanical behavior of beam structures in finite deformation including dissipative rate dependent mechanisms at constitutive level. The geometric and constitutive nonlinear dynamic response of several 2D and 3D structures was computed for different constitutive models including composites. Those examples show how the present formulation is able to capture different complex mechanical phenomena such as the uncoupling of the dynamic response from resonance due to inelastic incursions. Moreover, the present formulation which includes viscosity at material point level, suppress the high frequency content in the dynamic response which is a desirable characteristic of time stepping schemes. The study of a realistic flexible framed structure subjected to static and dynamic actions is also carried out. The fully coupled constitutive and geometric behavior of the frame is compared for both cases. A detailed study of the evolution of local damage indices, energy dissipation and ductility demands was presented.

\section{Acknowledgements}

This research was partially supported by CEE-FP6 Project FP6-505448 (GOCE), Risk Mitigation for Earthquakes and Landslides (LESSLOSS); by the Spanish Government: Ministerio de Fomento and Ministerio de Ciencia y Tecnología. Project Ref. MAT-2003-09768-C03-02, Delamination of reinforced matrix composites (DECOMAR); Ministerio de Educación y Ciencia. Project BIA200308700-C03-02, Numerical simulation of the seismic behavior of structures with energy dissipation devices. Project BIA2005-06952, Study of composite materials for design, reinforcement and retrofit of civil engineering structures (RECOMP). Project PSE 11-2005 (PSS-380000-2005-14) (HABITAT 2030). The International Center for Numerical Methods in Engineering (CIMNE) has also provided the support for this research. All this support is gratefully acknowledged. 


\section{Appendix A. Linearization of strain rates}

The linearized form of the material description of the reduced strain rate vectors given in Eqs. (4a) and (4b) can be obtained considering that $\Delta \hat{v}_{n}=\Delta \hat{\theta}-$ $\widetilde{\boldsymbol{v}}_{n} \Delta \hat{\theta}$ and $\Delta \tilde{\boldsymbol{v}}_{n}=\left(\Delta \tilde{\boldsymbol{\theta}}+\Delta \tilde{\boldsymbol{\theta}} \tilde{\boldsymbol{v}}_{n}-\tilde{\boldsymbol{v}}_{n} \Delta \tilde{\boldsymbol{\theta}}\right)$ in the following way:

$$
\begin{aligned}
\Delta \dot{\hat{\Gamma}}_{n}= & \Delta\left[\boldsymbol{\Lambda}^{\mathrm{T}}\left(\dot{\hat{\varphi}}, S-\widetilde{\boldsymbol{v}}_{n} \hat{\varphi}_{, S}\right)\right]=-\boldsymbol{\Lambda}^{\mathrm{T}} \Delta \tilde{\boldsymbol{\theta}}\left(\dot{\hat{\varphi}},_{S}-\widetilde{\boldsymbol{v}}_{n} \hat{\varphi}_{, S}\right) \\
& +\boldsymbol{\Lambda}^{\mathrm{T}} \Delta\left(\dot{\hat{\varphi}},_{S}-\tilde{\boldsymbol{v}}_{n} \hat{\varphi}_{, S}\right) \\
= & \boldsymbol{\Lambda}^{\mathrm{T}}\left(\boldsymbol{\Pi}\left[\dot{\hat{\varphi}},_{S}\right] \Delta \hat{\theta}+\widetilde{\boldsymbol{v}}_{n} \boldsymbol{\Pi}\left[\hat{\varphi}_{, S}\right] \Delta \hat{\theta}\right. \\
& \left.+\Delta \dot{\hat{\hat{\varphi}}}, \Delta-\Delta \widetilde{\boldsymbol{v}}_{n} \hat{\varphi}_{, S}-\widetilde{\boldsymbol{v}}_{n} \Delta \hat{\varphi}_{, S}\right) \\
= & \boldsymbol{\Lambda}^{\mathrm{T}}\left(\left(\boldsymbol{\Pi}\left[\dot{\hat{\varphi}}_{, S}\right]-\widetilde{\boldsymbol{v}}_{n} \boldsymbol{\Pi}\left[\hat{\varphi}_{, S}\right]\right) \Delta \hat{\theta}+\boldsymbol{\Pi}\left[\hat{\varphi}_{, S}\right] \Delta \dot{\hat{\theta}}\right. \\
& \left.+\Delta \dot{\hat{\hat{\varphi}}},-\widetilde{\boldsymbol{v}}_{n} \Delta \hat{\varphi}_{, S}\right),
\end{aligned}
$$

$$
\begin{aligned}
\Delta \dot{\widehat{\Omega}}_{n} & =0 \Delta\left[\boldsymbol{\Lambda}^{\mathrm{T}} \hat{v}_{n, S}\right]=-\boldsymbol{\Lambda}^{\mathrm{T}} \Delta \tilde{\boldsymbol{\theta}} \hat{v}_{n, S}+\boldsymbol{\Lambda}^{\mathrm{T}}\left(\Delta \tilde{\boldsymbol{v}}_{n, S}\right) \\
& =\boldsymbol{\Lambda}^{\mathrm{T}}\left[\Delta \dot{\hat{\theta}}_{, S}-\widetilde{\boldsymbol{v}}_{n} \Delta \hat{\theta}_{, S}\right] .
\end{aligned}
$$

The co-rotated linearized increment of the reduced strain rate vectors can be calculated considering the definition of the Lie's linearization, $\Delta[\bullet]=\boldsymbol{\Lambda}[\Delta(\bullet)][33,34]$ as follows:

$$
\begin{aligned}
& \Delta\left[\begin{array}{l}
\nabla \\
\hat{\gamma}_{n}
\end{array}\right]=\left(\boldsymbol{\Pi}\left[\dot{\hat{\varphi}}_{, S}\right]-\widetilde{\boldsymbol{v}}_{n} \boldsymbol{\Pi}\left[\hat{\varphi}_{, S}\right]\right) \Delta \hat{\theta}+\boldsymbol{\Pi}\left[\hat{\varphi}_{, S}\right] \Delta \dot{\hat{\theta}} \\
& +\Delta \dot{\hat{\varphi}}_{, S}-\widetilde{\boldsymbol{v}}_{n} \Delta \hat{\varphi}_{, S}, \\
& \Delta\left[\dot{\hat{\hat{\omega}}}_{n}\right]=\Delta \dot{\hat{\theta}}_{S}-\widetilde{\boldsymbol{v}}_{n} \Delta \hat{\theta}_{, S} .
\end{aligned}
$$

Finally, the material and co-rotated descriptions of the linearized increment of the strain rate vector existing on a material point on the beam cross-section are

$$
\begin{gathered}
\Delta \widehat{\mathscr{S}}_{n}=\Delta \dot{\hat{\mathscr{E}}}_{n}=\Delta \dot{\widehat{\Gamma}}_{n}+\Delta \dot{\widehat{\Omega}}_{n} \times \xi_{\beta} \widehat{E}_{\beta}, \\
\Delta \hat{S}_{n}=\Delta\left[\dot{\hat{\hat{\varepsilon}}}_{n}\right]=\boldsymbol{\Lambda} \Delta \widehat{\mathscr{S}}_{n}=\Delta\left[\dot{\hat{\hat{\gamma}}}_{n}\right]+\Delta\left[\dot{\hat{\hat{\omega}}}_{n}\right] \times \xi_{\beta} \hat{t}_{\beta} .
\end{gathered}
$$

The terms $\Delta \dot{\hat{\theta}}$ and $\Delta \dot{\hat{\theta}}_{, S}$ of Eqs. (A.3) and (A.4) can be expressed in term of $\Delta \hat{\theta}$ starting from $\Delta \widehat{V}_{n}=\Lambda^{\mathrm{T}} \Delta \hat{\theta}$ and considering the fact that Newmark's time stepping scheme allows to write $\Delta \widehat{V}_{n}=[\gamma /(\beta \Delta t)] \boldsymbol{\Lambda}^{\mathrm{T}} \mathbf{T}(\hat{\theta}) \Delta \hat{\theta}$ for the case dynamic variables belonging to the same tangent space, i.e. in the same iteration of the discrete version of the problem [49]. In this way, it is possible to write

$$
\Delta \dot{\hat{\theta}}=\frac{\gamma}{\beta \Delta t} \mathbf{T}(\hat{\theta}) \Delta \hat{\theta} ; \quad \Delta \dot{\hat{\theta}}_{, S}=\frac{\gamma}{\beta \Delta t}\left[\mathbf{T}(\hat{\theta}) \Delta \hat{\theta}_{, S}+\mathbf{T}(\hat{\theta})_{, S} \Delta \hat{\theta}\right]
$$

where the explicit forms of the tensors $\mathbf{T}(\hat{\theta})$ and $\mathbf{T}(\hat{\theta})_{{ }_{S}}$ can be consulted in Ref. [11]. Finally, Eqs. (A.3) and (A.4) can be rearranged as

$$
\begin{aligned}
& {\left[\begin{array}{cc}
\Delta\left[\dot{\hat{\hat{\gamma}}}_{n}^{\nabla}\right] \\
\Delta\left[\dot{\hat{\hat{\omega}}}_{n}^{\nabla}\right]
\end{array}\right]} \\
& =\underbrace{\left[\begin{array}{cc}
\left(\frac{\gamma}{\beta \Delta t}\left(\mathbf{I}-\tilde{\boldsymbol{v}}_{n}\right)\left[\frac{\mathrm{d}}{\mathrm{d} S}\right]\right) & \left.\left(\boldsymbol{\Pi}\left[\dot{\hat{\varphi}}_{, S}\right]-\tilde{\boldsymbol{v}}_{n} \boldsymbol{\Pi}[\hat{\varphi}]+\frac{\gamma}{\beta \Delta t} \boldsymbol{\Pi}\left[\hat{\varphi}_{, S}\right] \mathbf{T}\right)\right] \\
0 & \left(\frac{\gamma}{\beta \Delta t}\left\{\mathbf{T}\left[\frac{\mathrm{d}}{\mathrm{d} S}\right]+\mathbf{T}_{, S}\right\}-\tilde{\boldsymbol{v}}_{n}\left[\frac{\mathrm{d}}{\mathrm{d} S}\right]\right)
\end{array}\right]}_{[\hat{\gamma}(\hat{\varphi}, \hat{\theta})]} \\
& \quad \times\left[\begin{array}{c}
\Delta \hat{\varphi} \\
\Delta \hat{\theta}],
\end{array}\right.
\end{aligned}
$$

where $\mathbf{I}$ is the $3 \times 3$ identity matrix and the operator $\left[\frac{\mathrm{d}}{\mathrm{d} S}\right]$ is defined as $\left[\frac{\mathrm{d}}{\mathrm{d} S}\right](\bullet)=\mathbf{I} \frac{\mathrm{d}}{\mathrm{d} S}(\bullet)$. It is worth to note that the tensor $\mathscr{V}$ is configuration dependent and it couples the rotational and translational parts of the motion.

\section{References}

[1] S.S. Antman, Nonlinear Problems of Elasticity, Springer-Velag, 1991.

[2] J. Argyris, An excursion into large rotations, Comput. Methods Appl. Mech. Engrg. 32 (1982) 85-155.

[3] F. Armero, D. Ehrlich, Numerical modeling of softening hinges in the Euler-Bernoulli beams, Comput. Struct. 84 (2005) 641-656.

[4] F. Armero, D. Ehrlich, An analysis of strain localization and wave propagation in plastic models of beams at failure, Comput. Methods Appl. Mech. Engrg. 193 (2004) 3129-3171.

[5] F. Armero, D. Ehrlich, Finite element methods for the analysis of softening plastic hinges in beams and frames, Comput. Mech. 35 (2005) 237-264.

[6] F. Armero, I. Romero, Energy-dissipating momentum-conserving time-stepping algorithms for the dynamic of nonlinear Cosserat rods, Comput. Mech. 31 (2003) 3-26.

[7] A.H. Barbat, S. Oller, A. Hanganu, E. Oñate, Viscous damage model for Timoshenko beam structures, Int. J. Solids Struct. 34 (1997) 39533976.

[8] L. Briseghella, C.E. Majorana, C. Pellegrino, Conservation of angular momentum and energy in the integration of non-linear dynamic equations, Comput. Methods Appl. Mech. Engrg. 179 (1999) 247263.

[9] E. Car, S. Oller, E. Oñnate, An anisotropic elasto plastic constitutive model for large strain analysis of fiber reinforced composite materials, Comput. Methods Appl. Mech. Engrg. 185 (2000) 245-277.

[10] A. Cardona, A. Huespe, Evaluation of simple bifurcation points and post-critical path in large finite rotations problems, Comput. Methods Appl. Mech. Engrg. 175 (1999) 137-156.

[11] A. Cardona, M. Gerardin, A beam finite element non-Linear theory with finite rotations, Int. J. Numer. Methods Engrg. 26 (1988) 24032438.

[12] M.A. Crisfield, G. Jelenic̀, Objectivity of strain measures in the geometrically exact three-dimensional beam theory and its finiteelement implementation, Royal Soc. 455 (1999) 1125-1147.

[13] A.S. Elnashai, A.M. Mwafi, Overstrength and force reduction factors of multistorey reinforced concrete buildings, Struct. Des. Tall Build. 11 (2002) 329-351.

[14] C.A. Felippa, L.A. Crivelli, B. Haugen, A survey of the corecongruential formulation for geometrically nonlinear TL finite elements, Arch. Comput. Methods Engrg. 1 (1994) 1-48.

[15] F. Gruttmann, R. Sauer, W. Wagner, Theory and numerics of threedimensional beams with elastoplastic material behavior, Int. J. Numer. Methods Engrg. 48 (2000) 1675-1702.

[16] A.D. Hanganu, A.H. Barbat, E. Oñate, Metodología de Evaluación del Deterioro en Estructuras de Hormigoń Armado, Int. Center Numer. Methods Engrg. Monogr. 39 (1997). 
[17] A.D. Hanganu, E. Oñate, A.H. Barbat, Finite element methodology for local/global damage evaluation in civil engineering structures, Comput. Struct. 80 (2002) 667-1687.

[18] M. Iaura, S.N. Atluri, On a consistent theory, and variational formulation of finitely stretched and rotated 3-D space-curved beams, Comput. Mech. 4 (1989) 73-78.

[19] A. Ibrahimbegović, On finite element implementation of geometrically nonlinear Reissner's beam theory: three-dimensional curved beam elements, Comput. Methods Appl. Mech. Engrg. 122 (1995) 11-26.

[20] A. Ibrahimbegović, S. Mamouri, Energy conserving/decaying implicit time-stepping scheme for nonlinear dynamics of three-dimensional beams undergoing finite rotations, Comput. Methods Appl. Mech. Engrg. 191 (2002) 4241-4258.

[21] A. Ibrahimbegović, R.L. Taylor, H. Lim, Non-linear dynamics of flexible multibody systems, Comput. Struct. 81 (2003) 1113-1132.

[22] A. Ibrahimbegović, M. Al Mikdad, Quadratically convergent direct calculation of critical points for 3D structures undergoing finite rotations, Comput. Methods Appl. Mech. Engrg. 189 (2000) 107-120.

[23] A. Ibrahimbegović, S. Mamouri, R.L. Taylor, A.J. Chen, Finite element method in dynamics of flexible multibody systems: modeling of holonomic constraints and energy conserving integration schemes, Multibody Syst. Dyn. 4 (2000) 195-223.

[24] A. Ibrahimbegović, R.L. Taylor, On the role of frame-invariance in structural mechanics models at finite rotations, Comput. Methods Appl. Mech. Engrg. 191 (2002) 5159-5176.

[25] A. Ibrahimbegović, H. Shakourzadeh, J.L. Batoz, M.A. Mikdad, G. Ying-Qiao, On the role of geometrically exact and second-order theories in buckling and post-buckling analysis of three-dimensional beam structures, Comput. Struct. 61 (1996) 1101-1114.

[26] A. Ibrahimbegović, M. Al Mikdad, Finite rotations in dynamics of beams and implicit time-stepping schemes, Int. J. Numer. Methods Engrg. 41 (1998) 781-814.

[27] G. Jelenic, M. Saje, A kinematically exact space finite strain beam model-finite element formulation by generalized virtual work principle, Comput. Methods Appl. Mech. Engrg. 120 (1995) 131-161.

[28] A.J. Kappos, Seismic damage indices for RC buildings: Evaluation of concepts and procedures, Constr. Res. Commun. Ltd. 1 (1997) 78-87.

[29] J. Lubliner, On the thermodynamic foundations of non-linear mechanics, Int. J. Non-Linear Mech. 7 (1972) 237-254.

[30] R.K. Kapania, J. Li, On a geometrically exact curved/twisted beam theory under rigid cross-section assumption, Comput. Mech. 30 (2003) 428-443.

[31] R.K. Kapania, J. Li, A formulation and implementation of geometrically exact curved beam elements incorporating finite strains and finite rotations, Comput. Mech. 30 (2003) 444-459.

[32] L.E. Malvern, Introduction to the Mechanics of a Continuous Medium, Prentice-Hall, Englewood Cliffs, NJ, 1969.

[33] J. Mäkinen, H. Marjamäki, Total Lagrangian parametrization of rotation manifold, ENOC Hedinhoven Netherlands (2005) 522-530.

[34] J. Mäkinen, Critical study of the Newmark-scheme on manifold of finite rotation, Comput. Methods Appl. Mech. Engrg. 191 (2001) $817-828$.

[35] P. Mata A, S. Oller, A.H. Barbat, Static analysis of beam structures under nonlinear geometric and constitutive behavior, Comput. Methods Appl. Mech. Engrg. 196 (2007) 4458-4478.
[36] J. Oliver, M. Cervera, S. Oller, J. Lubliner, Isotropic damage models and smeared crack analysis of concrete, Proceedings 2nd ICCAADCS, vol. 2, Pineridge Press, Zell Am See, Austria, 1990, pp. 945958.

[37] J. Oliver, A.E. Huespe, Continuum approach to material failure in strong discontinuity settings, Comput. Methods Appl. Mech. Engrg. 193 (2004) 3195-3220.

[38] S. Oller, A.H. Barbat, Moment-curvature damage model for bridges subjected to seismic loads, Comput. Methods Appl. Mech. Engrg. 195 (2006) 4490-4511.

[39] S. Oller, E. Oñate, J. Miquel, S. Botello, A plastic damage constitutive model for composites materials, Int. J. Solids Struct. 33 (1996) 25012518.

[40] I. Papaioannou, M. Fragiadakis, M. Papadrakakis, Inelastic analysis of framed structures using the fiber approach, in: 5th GRACM International Congress on Computational Mechanics, 2005.

[41] M.S. Park, B.C. Lee, Geometrically non-linear and elastoplastic three-dimensional shear flexible beam element of Von-Mises-type hardening material, Int. J. Numer. Methods Engrg. 39 (1996) 383408.

[42] E. Reissner, On one-dimensional large-displacement finite-strain beam theory, Stud. Appl. Math. 2 (1973) LII 87-LII 95.

[43] E. Reissner, On one-dimensional finite-strain beam theory: the plane problem, J. Appl. Math. Phys. 23 (1972) 795-804

[44] I. Romero, F. Armero, An objective finite element formulation of the kinematics of geometrically exact rods and its use in the formulation of an energy-momentum conserving scheme in dynamics, Int. J. Numer. Methods Engrg. 54 (2002) 1683-1716.

[45] M. Saje, G. Turk, A. Kalagasidu, B. Vratanar, A kinematically exact finite element formulation of elastic-plastic curved beams, Comput. Struct. 67 (1998) 197-214.

[46] Y. Shao, S. Aval, A. Mirmiran, Fiber-element model for cyclic analysis of concrete-filled fiber reinforced polymer tubes, J. Struct. Engrg. 131 (2005) 292-303.

[47] J.C. Simo, A finite strain beam formulation. The three-dimensional dynamic problem. Part I, Comput. Methods Appl. Mech. Engrg. 49 (1985) 55-70.

[48] J.C. Simo, L. Vu-Quoc, A three-dimensional finite-strain rod model. Part II: Computational aspects, Comput. Methods Appl. Mech. Engrg. 58 (1986) 79-116.

[49] J.C. Simo, L. Vu-Quoc, On the dynamics in space of rods undergoing large motions - a geometrically exact approach, Comput. Methods Appl. Mech. Engrg. 66 (1988) 125-161.

[50] J.C. Simo, K.D. Hjelmstad, R.L. Taylor, Numerical formulations of elasto-viscoplastic response of beams accounting for the effect of shear, Comput. Methods Appl. Mech. Engrg. 42 (1984) 301330 .

[51] J.C. Simo, N. Tarnow, K.K. Wong, Exact energy-momentum conserving algorithms and symplectic schemes for nonlinear dynamics, Comput. Methods Appl. Mech. Engrg. 100 (1992) 63116.

[52] P. Betsch, P. Steinmann, Constrained dynamics of geometrically exact beams, Comput. Mech. 31 (2003) 49-59.

[53] M. Shugyo, Elastoplastic large deflection analysis of threedimensional steel frames, J. Struct. Engrg. ASCE (2003) 1259-1267. 